\title{
Synthesis of copper metal organic framework based on Schiff base tricarboxylate ligand for highly selective and sensitive detection of 2,4,6- trinitrophenol in aqueous medium
}

\section{Manpreet Kaur}

Punjabi University

M. Yususf

Punjabi University

Ashok Kumar Malik ( $\square$ malik_chem2002@yahoo.co.uk)

Punjabi University https://orcid.org/0000-0003-1045-6848

\section{Research Article}

Keywords: Schiff base, Metal-organic framework (MOF), Fluorescent sensor, Density functional theory (DFT), 2,4,6-Trinitrophenol (TNP), Photo-induced electron transfer (PET), Fluorescence resonance energy transfer (FRET).

Posted Date: July 20th, 2021

DOI: https://doi.org/10.21203/rs.3.rs-732172/v1

License: (c) (i) This work is licensed under a Creative Commons Attribution 4.0 International License. Read Full License 


\title{
Synthesis of copper metal organic framework based on Schiff base tricarboxylate ligand for highly selective and sensitive detection of 2,4,6-trinitrophenol in aqueous medium
}

\author{
Manpreet Kaur, M. Yusuf and Ashok Kumar Malik \\ Department of Chemistry, Punjabi University, Patiala-147002, Punjab, India
}

\begin{abstract}
By using Schiff base tricarboxylate ligand 5-(4-
\end{abstract} carboxybenzylideneamino)isophthalic acid $\left(\mathrm{H}_{3} \mathrm{CIP}\right)$, a new imine functionalized copper metal organic framework (MOF) has been synthesized solvothermally. It was fully characterized by Fourier Transform Infrared (FTIR) Spectroscopy, Powder X-Ray Diffraction (PXRD), Scanning Electron Microscopy (SEM), Energy Dispersive X-Ray Spectroscopy (EDS), and elemental mapping techniques. The as-synthesized MOF has been utilized as fluorescent probe for detection of nitro aromatic explosives (NAEs). The results show that the copper MOF can be developed into highly selective and sensitive sensor for detection of TNP in the aqueous medium via the "turn-off" quenching response. The linear fitting of the SternVolmer plot for TNP offered large quenching constant of $1.07 \times 10^{4} \mathrm{M}^{-1}$ for $\mathrm{Cu}-\mathrm{MOF}$ indicating the high sensitivity of the sensing process. Outstanding sensitivity of prepared material towards TNP detection was further validated by the low detection limit of $80 \mathrm{ppb}$ $(0.35 \mu \mathrm{M})$. The detailed mechanistic studies for their mode of action and density functional theory (DFT) calculations reveals that photo-induced electron transfer (PET) and fluorescence resonance energy transfer (FRET) processes, as well as electrostatic interactions (i.e. H-bonding) are the key factors for the turn-off response toward TNP by this fluorescent sensor. Thus, this new LMOF owing to their high water stability and remarkable functional features are potential candidates which can be developed into selective and sensitive TNP detection devices. 
Keywords: Schiff base; Metal-organic framework (MOF); Fluorescent sensor; Density functional theory (DFT); 2,4,6-Trinitrophenol (TNP); Photo-induced electron transfer (PET); Fluorescence resonance energy transfer (FRET) .

\section{GRAPHICAL ABSTRACT}

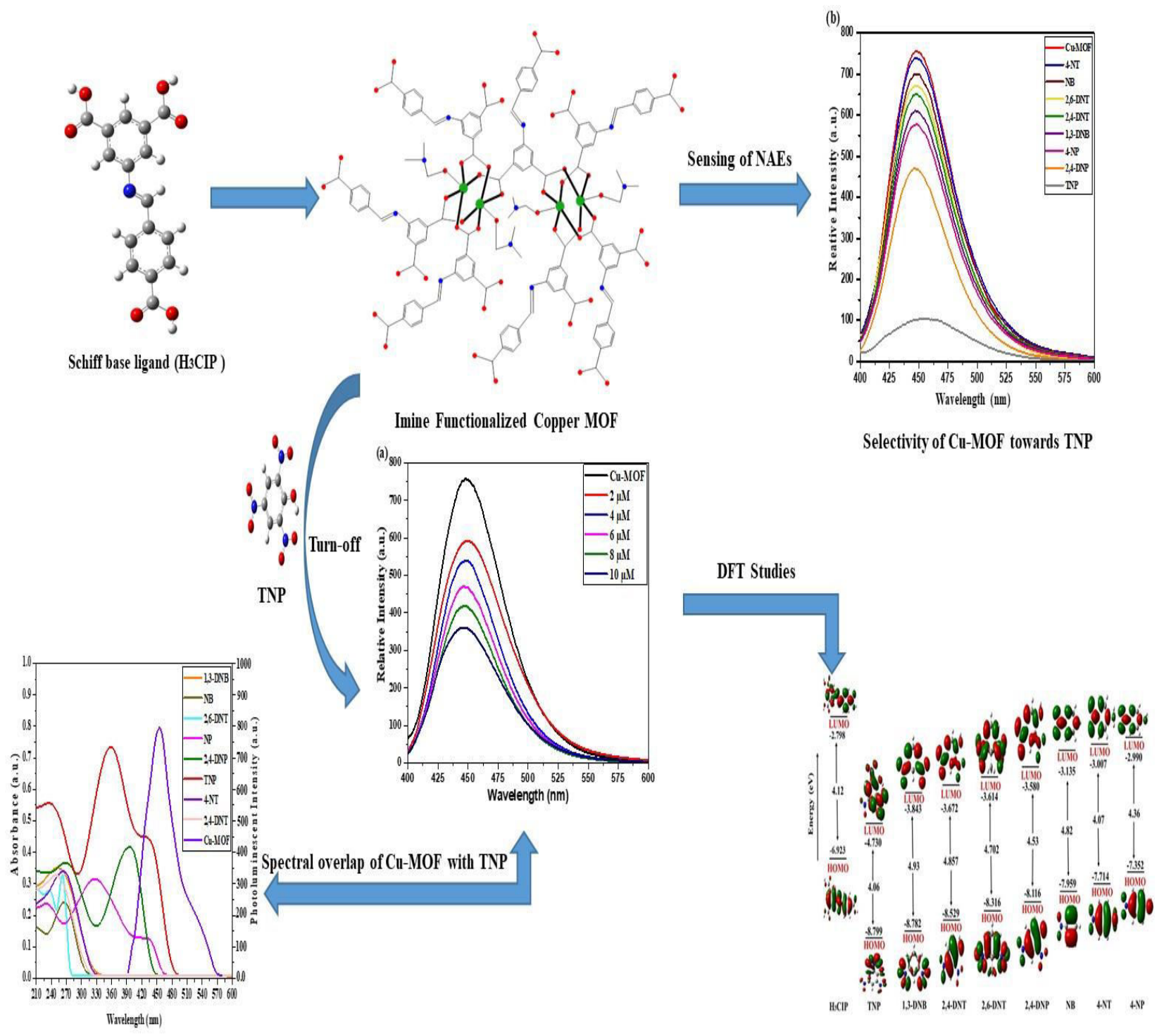




\section{HIGHLIGHTS}

- A Schiff base tricarboxylate ligand, 5-(4-carboxybenzylideneamino)isophthalic acid $\left(\mathrm{H}_{3} \mathrm{CIP}\right)$, has been synthesized using one step condensation method.

- Schiff base ligand has been utilized for the preparation of an imine functionalized copper MOF.

- Experiment showed that copper MOF showed excellent fluorescent properties.

- A fluorescent sensor showed the turn-off sensing response towards 2,4,6trinitrophenol.

- Fluorescence quenching of the host in the presence of guest like TNP can be explained through PET and FRET mechanisms.

- Experimental results are well supported by theoretical calculations. 


\section{INTRODUCTION}

Development of highly selective and sensitive sensing materials for the detection of hazardous nitro explosives (NEs) has become one of the most critical concerns all over the world because of ever increasing terrorist activities and unpredicted industrial disasters [1]. Both aromatic and aliphatic nitro compounds such as 2,4,6-trinitrotoluene (TNT), nitrobenzene (NB), 2,6-dinitrotoluene (2,6-DNT), 3,4-dinitrotoluene (3,4-DNT), 1,3dinitrobenzene (1,3-DNB), 2,4,6-trinitrophenol (TNP; also known as picric acid, PA), and nitro methane etc. are hazardous and/or explosive in nature. Among these NEs, picric acid is highly toxic and difficult to degrade. It is commonly used in various industries including dyes, polymers, plastics, pharmaceuticals, etc. [2]. The effluent released from these industries contains high concentration of TNP which gets easily accumulated in the soil and agricultural land owing to its high solubility in water [3]. Moreover, exposure to the high concentration of TNP can cause many health issues such as lung disorders, liver damage, asphyxiation, skin irritation, and even death [4]. Hence, precise, efficient, and selective detection of hazardous TNP in industrial waste, groundwater and soil is a significantly important [5]. Diverse analytical techniques e.g. mass spectrometry [6], high performance liquid chromatography (HPLC) [7], ion-mobility spectroscopy (IMS) [8], electrochemical

methods [9], and fluorescence spectroscopy [10] have been employed for the detection of TNP. However, among these fluorescence spectroscopy have gained tremendous attraction of the researchers because of its easy operation, low cost, good sensitivity/selectivity, and quick response time, etc. [11]. 
Till date, various types of fluorescent materials such as carbon dots [12], conjugated polymers [13], nanoparticles [14], and covalent organic frameworks [15] etc. have been developed for the detection of TNP. Thus, search on new materials for the selective and sensitive detection of TNP is still in high demand to solve the critical health and environmental issues.

In recent years, metal-organic frameworks (MOFs) draws special attention of researchers as fluorescent sensor for detection of TNP owing to their high porosity, high surface area, high surface to volume ratio, chemical functionalities, features of pore surface, well-defined crystalline structure, predictable interaction such as hydrogen bonding, $\pi-\pi$ stacking interactions, and coordination bonds between the guest species and MOF host [16]. Metal-organic frameworks (MOFs) are crystalline organic-inorganic hybrid materials constructed from the metal ions stitched together by the organic linker [17]. Such a kind of periodic porous crystal materials are considered to be promising candidates for many emerging applications such as gas storage/separation [18], heterogeneous catalysis [19], chemical sensing [20], drug delivery [21], and bio-imaging [22]. Particularly, the luminescent MOFs are tremendously attractive owing to their potential applications in chemical sensors and light-emitting devices [23]. The luminescent properties of MOF fluorescent sensors mainly depend upon the metal ions and organic ligands. A broad diversity of di- or multitopic N- and O-donor organic ligands have been widely utilized for the synthesis of MOFs. These ligands have significant influence on the chemical and physical properties of MOFs. Typically, the synthesis of di- or multi-topic N- and O-donor organic ligands involve multistep organic reactions, which could be costly and time consuming. With the increasing demand of time saving protocols and cheap starting reagents, different organic ligands have been searched in past few years. Schiff base ligands have gained enormous attention from the researchers due to cheap and readily available starting reagents (aldehyde/ ketones), less 
number of steps for their synthesis, typically one-step reaction, high yield, easy tuning of its properties by judicial selection of the reactants, stability, etc. [24]. The essential criteria for designing Schiff base ligand suitable for synthesis of MOF sensors for the detection of electron-deficient NEs are as follows: (i) the presence of $\pi$-electron-rich moiety in the molecule which can offer effective $\pi-\pi$ interactions with the electron-poor NEs, (ii) the presence of Lewis basic sites that can further form $\mathrm{H}$-bonding interactions with the nitrophenol derivatives.

Herein, considering these criteria, we have designed and synthesized Schiff base ligand 5-(4-carboxybenzylideneamino)isophthalic acid $\left(\mathrm{H}_{3} \mathrm{CIP}\right)$ with hydrophobic pendant moiety, namely, aromatic ring (benzene) and Lewis basic imine groups. In our design, the benzene ring provide effective $\pi-\pi$ stacking with the NAEs while the imine groups act as the Lewis basic recognition sites for the acidic NEs via the hydrogen bonding interactions $(\mathrm{H}-$ bonding). Interestingly, $\mathrm{H}_{3} \mathrm{CIP}$ has been utilized for preparation of copper MOF. This MOF has been found to show selective detection of TNP through a turn-off response with low detection limit of $80 \mathrm{ppb}(0.35 \mu \mathrm{M})$.

\section{EXPERIMENTAL}

\subsection{Materials and reagents}

4-Formylbenzoic acid (4-FBA), 5-aminoisophthalic acid (5-AIP), copper nitrate trihydrate $\left(\mathrm{Cu}\left(\mathrm{NO}_{3}\right)_{2} .3 \mathrm{H}_{2} \mathrm{O}\right)$, silica gel $\mathrm{G}$, and $\mathrm{N}, \mathrm{N}^{\prime}$-dimethylformamide (DMF) were purchased from Sigma Aldrich. NAEs: 4-nitrotoluene (4-NT), 2,4-dinitrotoluene (2,4-DNT), 2,6-dinitrotoluene (2,6-DNT), 1,3-dinitrobenzene (1,3-DNB), 2,4-dinitrophenol (2,4-DNP), 4-nitrophenol (4-NP), nitrobenzene (NB), and 2,4,6-trinitrophenol (TNP) were purchased 
from Avra synthesis Pvt. Ltd. All materials mentioned above were of analytical reagent grade and used without further purification.

\subsection{Measurements}

Fourier transform infrared spectra of the synthesized Schiff base ligand and copper MOF were scanned in the range $400-4000 \mathrm{~cm}^{-1}$ on a Perkin Elmer FT-IR Spectrophotometer. The progress of the aldimine condensation reaction was monitored by Thin-Layer Chromatography (TLC) using silica gel G (Sigma Aldrich). The spots on TLC plates were cautiously visualized by their exposure to the $\mathrm{I}_{2}$ fumes in the iodine chamber. Powder X-ray diffraction (PXRD) diffractograms for crystal structure determination were recorded using XPERT PRO Powder X-ray Diffractometer $\left[\mathrm{CuK}_{\alpha} \mathrm{X}\right.$-ray $(\lambda=1.5406 \AA), 1800 \mathrm{~W}(45 \mathrm{kV}, 40$ $\mathrm{mA})]$ in $2 \Theta$ range $2^{\circ}$ to $50^{\circ}$ keeping step size of $0.026^{\circ}$. JEOL, JSM-6510LV scanning electron microscope (SEM) was used for morphological and topographical analyses of synthesized MOF. Electron micrographs of gold coated sample were recorded at accelerating voltage of $15 \mathrm{kV}$ at a working distance of $11 \mathrm{~mm}$. EDS spectrum of gold coated sample rigidly mounted on the specimen stub was recorded using Oxford instrument (INCA X-act) EDS spectrometer equipped as an additional accessary to SEM. UV-Vis. spectrophotometer (Shimadzu UV-Vis 1600) was used for absorption studies. Shimadzu RF-5301PC spectrofluorometer was opted for photoluminescence studies. The theoretical studies were carried out using Gaussian 09 software.

\subsection{Methods}

\subsubsection{Synthesis of 5-(4-carboxybenzylideneamino)isophthalic acid $\left(\mathrm{H}_{3} \mathrm{CIP}\right)$}

In order to synthesize the copper MOF, an organic ligand 5-(4carboxybenzylideneamino)isophthalic acid $\left(\mathrm{H}_{3} \mathrm{CIP}\right)$ was first prepared by the aldimine condensation reaction of 5-aminoisophthalic acid (5-AIP) and 4-formylbenzoic acid (4-FBA) 
according to the synthesis method and conditions reported in literature [25]. The 5-AIP (1 mmol, $0.181 \mathrm{~g})$ and 4-FBA $(1 \mathrm{mmol}, 0.150 \mathrm{~g})$ were added to a round bottom flask $(100 \mathrm{~mL})$ containing $30 \mathrm{~mL}$ ethanol and slowly refluxed at $55{ }^{\circ} \mathrm{C}$ for $3 \mathrm{~h}$. The white precipitation with a Schiff base structure was filtered and washed by ethanol and diethyl ether several times, and then vacuum-dried.

\subsubsection{Synthesis of imine functionalized copper $M O F$}

The Schiff base tricarboxylate ligand 5-(4-carboxybenzylideneamino)isophthalic acid $\left(\mathrm{H}_{3} \mathrm{CIP}\right)(0.1 \mathrm{mmol}, 0.0313 \mathrm{~g})$ was dissolved (after sonication for 3 minutes) in $5 \mathrm{~mL} \mathrm{DMF}$ in a $20 \mathrm{~mL}$ glass vial and solid $\mathrm{Cu}\left(\mathrm{NO}_{3}\right)_{2} \cdot 3 \mathrm{H}_{2} \mathrm{O}(0.1 \mathrm{mmol}, 0.0241 \mathrm{~g})$ was added to this solution. The mixture was sonicated for $3 \mathrm{~min}$, sealed and then, heated in hot air oven at $100{ }^{\circ} \mathrm{C}$ for 24 h. During this period, light green crystalline material of copper MOF was formed. They were isolated by filtration, washed several times with DMF and diethylether followed by solvent exchange with methanol, and dried under vacuum.

\subsection{Luminescent sensing}

For luminescence sensing studies a stock solution was prepared by dissolving $1 \mathrm{mg}$ of finely grounded $\mathrm{Cu}-\mathrm{MOF}$ in $3 \mathrm{ml}$ water followed by sonication for $30 \mathrm{~min}$. The photoluminescent studies of above prepared stock solution was performed at room temperature. Inspired by its interesting fluorescent behavior, the potential applications of $\mathrm{Cu}-$ MOF as a fluorescence sensor was investigated for the detection of various NAEs. $20 \mu \mathrm{M}$ aqueous solution of each NAE (4-NT, NB, 2,4-DNT, 2,6-DNT, 1,3-DNB, 4-DNP, 4-NP, TNP) were prepared in water. For the selective studies, $1 \mathrm{mg}$ of MOF was added in $3 \mathrm{~mL}$ of stock solution of NAEs to produce the stable suspension. Resulting suspension was sonicated for $30 \mathrm{~min}$ before the photoluminescent (PL) studies. For the thorough study of selective sensing of TNP various concentration of analyte were prepared by dilution of $20 \mu \mathrm{M}$ 
solution. The PL spectra were recorded by spectrofluorometer keeping $\lambda_{\mathrm{ex}}=340 \mathrm{~nm}$ and 5 nm slit widths of both excitation and emission monochromators for all luminescent studies.

\subsection{Computational details}

The input file of $\mathrm{H}_{3} \mathrm{CIP}$ and various NAEs was prepared with Gauss view 5.08. All calculations were made using Gaussian 09. Theoretical calculations for the geometrical optimization, highest occupied molecular orbitals (HOMO) and lowest unoccupied molecular orbitals (LUMO) were performed at the density functional theory (DFT) levels using the B3LYP.

\section{Result and discussion}

\subsection{Synthesis and Characterization of Copper MOF.}

In the present work, 5-(4-carboxybenzylideneamino)isophthalic acid $\left(\mathrm{H}_{3} \mathrm{CIP}\right)$ was synthesized by the aldimine condensation reaction of an equimolar amount of 5aminoisophthalic acid and 4-formylbenzoic acid. $\mathrm{H}_{3}$ CIP, a Schiff base ligand, was then heated with $\mathrm{Cu}\left(\mathrm{NO}_{3}\right)_{2} \cdot 3 \mathrm{H}_{2} \mathrm{O}$ in $\mathrm{DMF}$ at $100{ }^{\circ} \mathrm{C}$ for $24 \mathrm{~h}$ to synthesize the copper MOF. The overall synthesis process was depicted in Scheme 1.

FTIR determinations were performed to establish the formation of Schiff base ligand, $\mathrm{H}_{3}$ CIP. The band observed around 1683 and $1688 \mathrm{~cm}^{-1}$ of 4-formylbenzoic acid (4-FBA) and 5-aminoisophthalic acid (5-AIP), respectively can be assigned to strong carboxylic $\mathrm{C}=\mathrm{O}$ antisymmetric stretching vibration are significantly blue shifted to $1725 \mathrm{~cm}^{-1}$ indicates the formation of $\mathrm{H}_{3}$ CIP ligand. The appearance of new peak at $1624 \mathrm{~cm}^{-1}$ which corresponding to the characteristic absorption of an imine group $(-\mathrm{CH}=\mathrm{N}-)$ can confirmed the formation of $\mathrm{H}_{3} \mathrm{CIP}$ ligand (Figure 1a). Meanwhile, the $\mathrm{C}-\mathrm{H}$ vibration peak of meta-disubstituted benzene ring blue-shifts from $748 \mathrm{~cm}^{-1}$ for 5-AIP to $767 \mathrm{~cm}^{-1}$ for $\mathrm{H}_{3} \mathrm{CIP}$ [26]. The very small shifts of both $\mathrm{C}=\mathrm{O}$ antisymmetric stretch peak and $\mathrm{C}-\mathrm{H}$ vibration peak of meta-disubstituted benzene 
ring as well as appearance of $-\mathrm{CH}=\mathrm{N}$ - vibration peak confirmed the formation of $\mathrm{H}_{3} \mathrm{CIP}$ ligand. FTIR analysis was further utilized to provide the conclusive evidence of formation of copper MOF from $\mathrm{H}_{3} \mathrm{CIP}$. The strong peak at $1725 \mathrm{~cm}^{-1}$ which corresponding to the characteristic antisymmetric stretch absorption of carbonyl of $-\mathrm{COOH}$ group, present in the $\mathrm{H}_{3} \mathrm{CIP}$ disappear in the copper MOF (Figure 1b). The new strong bands appeared at 1379 and $1637 \mathrm{~cm}^{-1}$ in the FTIR spectrum of the copper MOF can be regarded as the symmetric and antisymmetric stretching modes of the coordinated (-COO) group, respectively. It indicates that $-\mathrm{COOH}$ of $\mathrm{H}_{3} \mathrm{CIP}$ involved in the coordination with copper. It should be noted that the values of $\Delta v\left(\Delta v=v_{\text {as }}(\mathrm{COO})-v_{\mathrm{s}}(\mathrm{COO})\right)$ for metal carboxylates are useful tool for distinguish the coordination modes. According to this, the carboxylate group coordinates in a bidentate chelating fashion if $\Delta v(\mathrm{COO})$ is significantly less compared to that of the ligand; a monodentate fashion if $\Delta v(\mathrm{COO})$ of the complex is much larger compared to that of the ligand; and bridging can be reflected when near to the ionic values [27]. Therefore, the observed difference in antisymmetric and symmetric carbonyl stretching frequencies $\Delta v$ for $\mathrm{Cu}-\mathrm{MOF}$ was $251(\Delta v=1637-1379=250)$ significantly less than $\mathrm{H}_{3} \mathrm{CIP}(\Delta v=1725-1288$ $=378$ ), indicating the chelating bidentate coordination mode of carboxylate moieties in copper MOF. The peak at $1590 \mathrm{~cm}^{-1}$ peak can be attributed to aromatic rings $\mathrm{C}=\mathrm{C}$ stretching vibration in the copper MOF. The vibrations associated with metal linker bond are present below $600 \mathrm{~cm}^{-1}$. These vibrational modes are difficult to observe with IR method. The bonding between $\mathrm{Cu}-\mathrm{O}$ appears at $491 \mathrm{~cm}^{-1}$, which is another conclusive evidence of coordination between Schiff base ligand and $\mathrm{Cu}^{2+}[28]$. The absence of peaks at 410, 500, 610 , and $615 \mathrm{~cm}^{-1}$ indicates that synthesized material is free from $\mathrm{CuO}$ and $\mathrm{Cu}_{2} \mathrm{O}$ during the nucleation of copper MOF. These results indicated that high purity copper MOF was synthesized successfully. 
PXRD analysis was done to examine the crystallinity and phase purity of synthesized copper MOF. It has been already reported in literature that when the ligands possessing the isophthalic acid moiety are employed in $\mathrm{Cu}^{2+}$ chemistry, they often afford structures which are similar to that of HKUST-1 [29]. Herein, $\mathrm{Cu}-\mathrm{MOF}$ has been prepared using $\mathrm{H}_{3} \mathrm{CIP}$ ligand which is significantly extended ligand compared to other commonly used tricarboxylic acids (for example, trimesic acid which was used for the preparation of $\mathrm{Cu}-\mathrm{BTC}$ or HKUST-1 MOF) and possesses the semi-rigid $\mathrm{C}=\mathrm{N}$ moiety. Furthermore, $\mathrm{H}_{3} \mathrm{CIP}$ ligand is similar to the $\mathrm{PEIPH}_{2}$ Schiff base ligand but contains benzoic acid moiety in place of pyridyl group [29]. Thus, it was found that the PXRD pattern of as-synthesized Cu-MOF was very similar to the simulated pattern reported for the Cu-BTC (HKUST-1) as well as Cu-PEIP (whose structure similar to HKUST-1) confirming the formation of isostructural MOF [28]. The PXRD pattern of the synthesized sample is shown in Figure 2 which displays the main diffraction peaks of $\mathrm{Cu}-\mathrm{MOF}$ at $2 \theta=5.912^{\circ}, 7.121^{\circ}, 9.811^{\circ}, 11.904^{\circ}, 12.414^{\circ}, 15.411^{\circ}, 16.908^{\circ}, 18.021^{\circ}$, $19.615^{\circ}, 26.578^{\circ}$, and $29.573^{\circ}$ corresponding to the Miller indices (111), (200), (220), (222), (400), (331), (422), (511), (440), (731), and (751), respectively [28]. The characteristic diffraction peaks of $\mathrm{Cu}_{2} \mathrm{O}$ at $2 \theta=36.4^{\circ}, 42.3^{\circ}$, and $43.3^{\circ}$ are not observed in the PXRD pattern of the synthesized sample indicates the high purity of synthesized copper MOF product [30]. The crystalline nature of the synthesized material is indicated from the sharp observed PXRD peaks. Unfortunately, all efforts used for the preparation of single crystal of copper MOF from $\mathrm{H}_{3} \mathrm{CIP}$ ligand such as use of series of modulators (like acetic acid, benzoic acid) in varying concentrations and mixture of solvents has been failed. Based on the FTIR and PXRD analysis, the structure of as-synthesized imine functionalized MOF has been proposed which resembles the structure of HKUST-1 and Cu-PEIP MOF as shown in Figure 3. 
SEM images were taken to reveal the surface morphology of the synthesized $\mathrm{Cu}$ MOF. SEM image of copper MOF are shown in Figure 4. The SEM of copper MOF displayed the flake like morphology (Figure $4 \mathrm{a}$ and $4 \mathrm{~b}$ ). Zoomed images indicated uniform sphere like particles (Figure 4c). EDS was carried out to investigate the elemental composition of copper MOF. EDS of spectra of copper MOF are shown in Figure 5. Compositional analysis carried from EDS spectra reveal the prescence of carbon (C), nitrogen $(\mathrm{N})$, oxygen $(\mathrm{O})$, and copper $(\mathrm{Cu})$ with detected weights (atomic ratios) to be $44.82 \%(60.40 \%), 6.36 \%(7.35 \%)$ and $22.63 \%(5.76 \%)$, respectively. In the recorded EDS spectra an extra peak at $2.2 \mathrm{keV}$ is also observed due to coating of sample by gold for conductive SEM analysis. Presence and distribution of these elements was further confirmed with the elemental maps of $\mathrm{Cu}, \mathrm{C}, \mathrm{N}$, and $\mathrm{O}$ in the Figure 6(a-d). EDS analysis confirms the formation of high purity copper MOF by illustrating the prescence of elements $(\mathrm{C}, \mathrm{O}, \mathrm{N}$, and $\mathrm{Cu}$ ) of the organic ligand and metal in the final structure of MOF.

\subsection{Water stability}

For the successful in-field applications, the stability of MOFs towards water, moisture, and humidity is crucial. Thus, we have assessed the impact of water on the structural integrity of synthesized $\mathrm{Cu}-\mathrm{MOF}$. Typically, $\mathrm{Cu}-\mathrm{MOF}$ was soaked in water for a period of $24 \mathrm{~h}$ and then analyzed by PXRD analysis. Furthermore, to understand the impact of TNP on the stability of Cu-MOF, it was also soaked in aqueous TNP solutions for $24 \mathrm{~h}$. PXRD analyses indicated that the crystallinity of $\mathrm{Cu}-\mathrm{MOF}$ was not destroyed even after soaking in water or aqueous TNP solution for $24 \mathrm{~h}$.

\subsection{Photoluminescent properties}

Luminescent properties of $\mathrm{H}_{3} \mathrm{CIP}$ and $\mathrm{Cu}-\mathrm{MOF}$ was explored in water at room temperature. As shown in Figure 7, the excitation spectrum of ligand $\mathrm{H}_{3} \mathrm{CIP}$ presents a broad 
peak centered at $355 \mathrm{~nm}$. Upon excitation at $355 \mathrm{~nm}, \mathrm{H}_{3} \mathrm{CIP}$ display a broad emission peak centered at $423 \mathrm{~nm}$, which may arise from a $\pi-\pi^{*}$ or $n-\pi^{*}$ electron transition within the ligand. However, as seen from Figure 7, the emission spectrum of $\mathrm{Cu}-\mathrm{MOF}$ displays a maximum emission at $450 \mathrm{~nm}$ upon excitation at $340 \mathrm{~nm}$, a significant red shift in comparison with the $\mathrm{H}_{3} \mathrm{CIP}$ ligand. This may be due to the coordination interactions within the framework, which may increase the rigidity of the ligands, decrease the energy loss, and in this manner effect the electron transition.

\subsection{Detection of TNP}

To investigate the sensing properties of $\mathrm{Cu}-\mathrm{MOF}$ toward different NAEs, water was selected as the solvent because $\mathrm{Cu}-\mathrm{MOF}$ shows high stability, good dispersion, and excellent luminescent properties in water. Figure 8a shows the emission spectra of $\mathrm{Cu}-\mathrm{MOF}$ dispersed in water at different excitation wavelengths from $260 \mathrm{~nm}$ to $360 \mathrm{~nm}$ with maximum emission intensity observed at $757 \mathrm{~nm}$ at $\lambda_{\mathrm{ex}}=340 \mathrm{~nm}$. Thus, $340 \mathrm{~nm}$ excitation wavelength has been selected as appropriate excitation wavelength for the further PL studies to detect NAEs. A $1 \mathrm{mg}$ of powdered sample of as-synthesized $\mathrm{Cu}-\mathrm{MOF}$ was ground and introduced into the $3 \mathrm{~mL}$ aqueous solutions of $20 \mu \mathrm{M}$ of different NAEs (TNP, NB, 4-NT, 4-NP, 2,4DNP, 2,4-DNT, 2,6-DNT, and 1,3-DNB) to form stable suspension after ultrasonication for $30 \mathrm{~min}$. The luminescent response of suspensions were recorded and compared in Figure $8 \mathrm{~b}$. The results revealed that all examined NAEs quenched the luminescent intensity of $\mathrm{Cu}-\mathrm{MOF}$. It is worth mentioning that the contacting between TNP and $\mathrm{Cu}-\mathrm{MOF}$ leads to the significant suppression effect on the luminescence, while other NAEs perform slight influence on the emission intensity of the Cu-MOF. As depicted from Figure 8c, the histogram of luminescence intensity of $\mathrm{Cu}-\mathrm{MOF}$ around $450 \mathrm{~nm}$ shows that after immersing into $20 \mu \mathrm{M}$ aqueous solution of TNP, the seven fold quenching of emission intensity was observed compared to original one. The fluorescence quenching efficiency was calculated by $\left[\left(\mathrm{I}_{0}-\right.\right.$ 
$\left.\mathrm{I}) / \mathrm{I}_{0}\right] \times 100 \%$, where $\mathrm{I}_{0}$ and $\mathrm{I}$ are the fluorescence intensities before and after addition of the analyte. In particular, it has been observed that TNP addition into $\mathrm{Cu}-\mathrm{MOF}$ aqueous suspensions shows highest fluorescence quenching efficiency amounting to $86.12 \%$ compared to 2,4-DNP ( 38.04\%), 4-NP ( 23.64 \%), 1,3-DNB ( 19.2 \%), 2,4-DNT $(\sim 14.13 \%), 2,6-\mathrm{DNT}(\sim 11.36 \%), \mathrm{NB}(\sim 7.52 \%)$, and 4-NT $(\sim 2.24 \%)$ as shown in Figure 8d. These outcomes implied that the $\mathrm{Cu}-\mathrm{MOF}$ could operate as a "turn-off" fluorescent sensor for TNP.

To understand the fluorescence quenching response of $\mathrm{Cu}-\mathrm{MOF}$ towards TNP, we measured the luminescent responses of $1 \mathrm{mg}$ of $\mathrm{Cu}-\mathrm{MOF}$ immersed in TNP aqueous solutions with different concentrations. It is obvious that the luminescence intensity of $\mathrm{Cu}$ MOF incorporated with TNP shows a strong dependence on the concentration of TNP. As depicted from Figure 9a, the emission intensity of $\mathrm{Cu}-\mathrm{MOF}$ decreases gradually with the increase of TNP concentration in the range from 1 to $10 \mu \mathrm{M}$. Quantatively, the quenching effect of TNP can be analyzed and calculated by the Stern-Volmer equation:

$$
\mathrm{I}_{0} / \mathrm{I}=1+\mathrm{K}_{\mathrm{SV}}[\mathrm{TNP}]
$$

where, $\mathrm{I}_{0}$ and $\mathrm{I}$ are the luminescent intensity of the $\mathrm{Cu}-\mathrm{MOF}$ suspension at $450 \mathrm{~nm}$ in the presence and absence of TNP, $\mathrm{K}_{\mathrm{SV}}$ is the Stern-Volmer quenching constant $\left(\mathrm{M}^{-1}\right)$, and [TNP] is the concentration of TNP. As seen from Figure 9b, there is a good linear relationship (correlation coefficient $\mathrm{R}^{2}=0.9962$ ) between $\mathrm{I} / \mathrm{I}_{0}$ and $[\mathrm{TNP}]$ at a low concentration range of $0-10 \mu \mathrm{M}$, indicating that the quenching effect of TNP on the luminescence of $\mathrm{Cu}-\mathrm{MOF}$ agrees the Stern-Volmer mode well. The Stern-Volmer quenching constant value is calculated to be $1.07 \times 10^{4} \mathrm{M}^{-1}$, which reveals a strong quenching effect on the $\mathrm{Cu}-\mathrm{MOF}$ luminescence Figure 9c. The limit of detection (LOD) of $\mathrm{Cu}-\mathrm{MOF}$ towards TNP was calculated using the equation $\operatorname{LOD}=3.3 \sigma / \mathrm{m}$, where $\sigma=$ standard deviation and $\mathrm{m}=$ 
slope of the linear curve plotted at lower concentration for DL calculations. The LOD values for TNP was found to be $80 \mathrm{ppb}(0.35 \mu \mathrm{M})$. The $\mathrm{K}_{\mathrm{sv}}$ and LOD values demonstrate $\mathrm{H}_{2} \mathrm{~L}$ are good fluorescent sensor for detection of TNP.

In order to perform the time dependent detection of TNP, $1 \mathrm{mg}$ of $\mathrm{Cu}-\mathrm{MOF}$ was introduced into the $3 \mathrm{~mL}$ of $20 \mu \mathrm{M}$ TNP aqueous solution and emission spectra were recorded with $1 \mathrm{~min}$ intervals up to $10 \mathrm{~min}$ upon excitation at $340 \mathrm{~nm}$. It can be noticed from Figure 10a, the luminescence emission reached a constant value after $1 \mathrm{~min}$. Along with the contact time between TNP and $\mathrm{Cu}-\mathrm{MOF}$, the emission intensity of the suspension remains almost unchanged, which tells that TNP induced fluorescence weaken reaction is relatively fast. Hence, the presented material could be utilized for the rapid detection of TNP.

Inspired by the highest fluorescence quenching efficiency of TNP among the selected NAEs, the specificity of $\mathrm{Cu}-\mathrm{MOF}$ towards TNP in the co-existence of other NAEs was examined to analyze the competing effect of NAEs. In such an experiment, the fluorescence intensity of $\mathrm{Cu}-\mathrm{MOF}$ was recorded by the $3 \mathrm{~mL}$ addition of a $20 \mu \mathrm{M}$ aqueous solution of particular nitro analyte followed by the $3 \mathrm{~mL}$ addition of $20 \mu \mathrm{M}$ aqueous solution of TNP. As shown in Figure 10b, the insignificant change in the intensity was observed in the presence of other NAEs followed by tremendous decrease in intensity after the TNP addition, whereas in the presence of 2,4-DNP and 4-NP, significant decrease was observed followed by moderate change after the TNP addition. These experimental results proved that the existence of other explosives would not disturb the detection of TNP. Hence, it becomes obvious that the presented material has remarkable selectivity towards TNP over other congeners.

\subsection{Possible mechanism for the TNP detection}

For understanding the high selectivity of $\mathrm{Cu}-\mathrm{MOF}$ towards TNP, the sensing mechanism was examined. The possible mechanisms of turn-off quenching between electron- 
rich $\mathrm{Cu}-\mathrm{MOF}$ and electron-deficient TNP are: (1) Destruction of crystalline structure of MOF; (2) Photo-induced electron transfer (PET); (3) Fluorescence resonance energy transfer (FRET); and (4) Molecular interactions (such as electrostatic and $\pi-\pi$ interactions). The PXRD pattern, and FTIR of copper MOF (Figure 11) still maintained intact before and after quenched by TNP. These results confirmed that the interactions between TNP and copper MOF could result in the quench phenomenon, but it was not strong enough to destroy the crystalline structure of copper MOF.

As proposed for many other MOFs, the PET might govern the highly selective detection of NAEs by the Cu-MOF. Generally, the LUMOs of the electron-deficient NAEs are situated between the conduction band (CB) and valence band (VB) of the MOFs [31]. Therefore, an electron transfer process can easily take place from the $\mathrm{CB}$ of $\mathrm{Cu}-\mathrm{MOF}$ to the LUMO of electron-deficient NAEs. This process can cause quenching of the fluorescence intensity of $\mathrm{Cu}-\mathrm{MOF}$ upon excitation. To validate the PET mechanism relevant for this turnoff quenching, the $\mathrm{E}_{\mathrm{HOMO}}$ and $\mathrm{E}_{\mathrm{LUMO}}$ energies of the Schiff base tricarboxylate ligand $\left(\mathrm{H}_{3} \mathrm{CIP}\right)$ and NAEs were calculated using DFT with the basis set B3LYP/6-311G and the Gaussian 09 package program (Figure 12). The existence of easy electron transition between $\mathrm{E}_{\mathrm{HOMO}}$ and $\mathrm{E}_{\mathrm{LUMO}}$ of reacting species was established by the popular Frontier Molecular Orbital (FMO) theory. According to FMO theory, the quenching efficiency of analyte species is a function of its $\mathrm{E}_{\mathrm{HOMO}}$ and $\mathrm{E}_{\mathrm{LUMO}}$. It is well known that the species with high energy HOMO are more capable to donate electrons than those with lower energy HOMO. On the other hand, species with low-lying LUMO are more capable to accept electrons than those with higher energy LUMO and thus will show higher quenching efficiency. From the HOMO-LUMO energy gaps, it is inferred that PET takes place from the HOMO of the $\mathrm{Cu}-\mathrm{MOF}$ to the LUMO of electron-deficient TNP compared to other NAEs. This agrees well with the observed maximum quenching efficiency for TNP. It was observed that TNP molecule has the lowest 
LUMO energy value $(-4.730 \mathrm{eV})$. Thus, it is concluded that the higher quenching efficiency of TNP. The HOMO and LUMO orbital energies for the NAEs are presented in Figure 12. The ascending order for the calculated LUMO energy values of the NAEs is: TNP $<1,3-$ DNB $<2,4-\mathrm{DNT}<2,6-\mathrm{DNT}<2,4-\mathrm{DNP}<\mathrm{NB}<4-\mathrm{NT}<4-\mathrm{NP}$. The ease of electron transfer from the electron-rich $\mathrm{Cu}-\mathrm{MOF}$ to the electron-deficient NAEs should follow reverse of this sequence. Therefore, the highest quenching efficiency of TNP agrees well with its lowest LUMO energy value. Moreover, the trend of quenching efficiencies (i.e., TNP > 2,4-DNP > 4-NP > 1,3-DNB > 2,4-DNT > 2,6-DNT > NB > 4-NT) is not fully in accordance with the LUMO energy values of other analytes. These theoretical results are not in good correlation in our experimental results. These results suggest that only PET mechanism cannot completely account for the observed quenching behavior. Moreover, FRET mechanism might be recognized for the quenching process.

In order for FRET to take place, there should be an efficient spectral overlap between the emission spectrum of the fluorophore and the absorption spectrum of the analyte. As presented in Figure 13, TNP shows a strong absorption band at $353 \mathrm{~nm}$ and copper MOF exhibits a strong fluorescence emission band at $471 \mathrm{~nm}$. There is a large overlap between the absorption spectrum of TNP and the emission spectrum of copper MOF. On the other hand, other analytes have almost no such spectral overlap. The largest spectral overlap for TNP is in accordance with its highest quenching efficiency. The spectral overlap supports the fact that the fluorescence quenching by TNP takes place by an energy transfer mechanism.

In addition, the fluorescence quenching might be ascribed to some molecular interactions such as electrostatic (between the hydroxyl group of electron-deficient TNP and the basic sites i.e. imine group of electron-rich copper MOF) and $\pi-\pi$ interactions (between the benzene rings of TNP and copper MOF) (Scheme 2). In order to verify that the electrostatic interactions are involved in the fluorescence quenching process, the quenching 
efficiencies of some NAEs containing one hydroxyl group, such as TNP, 2,4-DNP and PNP were compared. The quenching efficiencies decreased in the sequence: TNP > 2,4-DNP > PNP, which is in accordance with the acidity of the phenolic protons. A decrease in the quenching efficiency with the reduction in the acidity can also be ascribed to the presence of imine groups where the imine groups can form H-bonding interactions with the phenolic protons of the nitrophenol derivatives, which are absent in the other NAE analytes. A probable mechanism for the selective sensing of TNP from these observations has been illustrated in Scheme 2, where an energy transfer via $\pi-\pi$ interactions between TNP and benzene moieties and the above mentioned H-bonding with the more acidic phenolic proton of TNP play important roles. This suggests that electron and energy transfer processes as well as H-bonding processes interplay together for the observed quenching response.

\section{CONCLUSIONS}

In summary, we have successfully synthesized a luminescent MOF $(\mathrm{Cu}-\mathrm{MOF})$ with an imine functionalized tricarboxylate ligand. $\mathrm{Cu}-\mathrm{MOF}$ show outstanding photoluminescent properties and remarkable water stability even when soaked in water for $24 \mathrm{~h}$. These features

of $\mathrm{Cu}-\mathrm{MOF}$ have been exploited to develop an excellent approach for water based fluorescence quenching detection of TNP. Results showed LODs is as low as $80 \mathrm{ppb}(0.35$ $\mu \mathrm{M})$ and Stern-Volmer quenching constants as high as $1.07 \times 10^{4} \mathrm{M}^{-1}$ for aqueous phase detection of TNP. The as-synthesized $\mathrm{Cu}-\mathrm{MOF}$ exhibited outstanding sensitivity, and high selectivity toward TNP without suffering from the interference of other NAEs. The highest quenching efficiency of TNP among several other NAEs can be related to the existence of electron and energy transfer processes as well as molecular interactions ( $\pi-\pi$ and electrostatic) between the MOF and TNP. Eventually, the present study provides the new insight into the design of water stable LMOF-based explosive sensors, which may be useful under more 
realistic conditions for the detection of TNP which has relevance to homeland security and ecological concerns. 


\section{References}

[1] M. Shyamal, P. Mazumdar, S. Maity, S. Samanta, G.P. Sahoo, A. Misra, Highly selective turn-on fluorogenic chemosensor for robust quantification of $\mathrm{Zn}$ (II) based on aggregation induced emission enhancement feature, ACS Sensors 1(6) (2016) 739-747.

[2] B. Parmar, Y. Rachuri, K.K. Bisht, R. Laiya, E. Suresh, Mechanochemical and conventional synthesis of $\mathrm{Zn}$ (II)/Cd (II) luminescent coordination polymers: dual sensing probe for selective detection of chromate anions and TNP in aqueous phase, Inorganic chemistry 56(5) (2017) 2627-2638.

[3] G. Chakraborty, S.K. Mandal, Design and development of fluorescent sensors with mixed aromatic bicyclic fused rings and pyridyl groups: solid mediated selective detection of 2, 4, 6trinitrophenol in water, ACS omega 3(3) (2018) 3248-3256.

[4] S. Maity, M. Shyamal, D. Das, P. Mazumdar, G.P. Sahoo, A. Misra, Aggregation induced emission enhancement from antipyrine-based schiff base and its selective sensing towards picric acid, Sensors and Actuators B: Chemical 248 (2017) 223-233.

[5] B. Parmar, Y. Rachuri, K.K. Bisht, E. Suresh, Syntheses and structural analyses of new 3D isostructural $\mathrm{Zn}$ (II) and Cd (II) luminescent MOFs and their application towards detection of nitroaromatics in aqueous media, ChemistrySelect 1(19) (2016) 6308-6315.

[6] L. Barron, E. Gilchrist, Ion chromatography-mass spectrometry: a review of recent technologies and applications in forensic and environmental explosives analysis, Analytica chimica acta 806 (2014) 27-54.

[7] M. Rahimi-Nasrabadi, M.M. Zahedi, S.M. Pourmortazavi, R. Heydari, H. Rai, J. Jazayeri, A. Javidan, Simultaneous determination of carbazole-based explosives in environmental waters by dispersive liquid-liquid microextraction coupled to HPLC with UV-Vis detection, Microchimica Acta 177(1) (2012) 145-152. 
[8] P. Guerra-Diaz, S. Gura, J.R. Almirall, Dynamic planar solid phase microextraction- ion mobility spectrometry for rapid field air sampling and analysis of illicit drugs and explosives, Analytical chemistry 82(7) (2010) 2826-2835.

[9] F. Yan, Y. He, L. Ding, B. Su, Highly ordered binary assembly of silica mesochannels and surfactant micelles for extraction and electrochemical analysis of trace nitroaromatic explosives and pesticides, Analytical chemistry 87(8) (2015) 4436-4441.

[10] P. D Jawale Patil, R. D Ingle, S. M Wagalgave, R. S Bhosale, S. V Bhosale, R. P Pawar, S. V Bhosale, A naphthalimide-benzothiazole conjugate as colorimetric and fluorescent sensor for selective trinitrophenol detection, Chemosensors 7(3) (2019) 38.

[11] W. Wang, H. Li, M. Yin, K. Wang, Q. Deng, S. Wang, Y. Zhang, Highly selective and sensitive sensing of 2, 4, 6-trinitrophenol in beverages based on guanidine functionalized upconversion fluorescent nanoparticles, Sensors and Actuators B: Chemical 255 (2018) $1422-1429$.

[12] A.B. Siddique, A.K. Pramanick, S. Chatterjee, M. Ray, Amorphous carbon dots and their remarkable ability to detect 2, 4, 6-trinitrophenol, Scientific reports 8(1) (2018) 1-10.

[13] S. Jiang, L. Meng, W. Ma, Q. Qi, W. Zhang, B. Xu, L. Liu, W. Tian, Morphology controllable conjugated network polymers based on AIE-active building block for TNP detection, Chinese Chemical Letters 32(3) (2021) 1037-1040.

[14] M. Rong, L. Lin, X. Song, T. Zhao, Y. Zhong, J. Yan, Y. Wang, X. Chen, A label-free fluorescence sensing approach for selective and sensitive detection of 2, 4, 6-trinitrophenol (TNP) in aqueous solution using graphitic carbon nitride nanosheets, Analytical chemistry 87(2) (2015) 1288-1296.

[15] M.-W. Zhu, S.-Q. Xu, X.-Z. Wang, Y. Chen, L. Dai, X. Zhao, The construction of fluorescent heteropore covalent organic frameworks and their applications in spectroscopic 
and visual detection of trinitrophenol with high selectivity and sensitivity, Chemical Communications 54(18) (2018) 2308-2311.

[16] S. Nandi, S. Biswas, A recyclable post-synthetically modified Al (iii) based metalorganic framework for fast and selective fluorogenic recognition of bilirubin in human biofluids, Dalton Transactions 48(25) (2019) 9266-9275.

[17] D. Zhao, Y. Cui, Y. Yang, G. Qian, Sensing-functional luminescent metal-organic frameworks, CrystEngComm 18(21) (2016) 3746-3759.

[18] H. Li, L. Li, R.-B. Lin, W. Zhou, Z. Zhang, S. Xiang, B. Chen, Porous metal-organic frameworks for gas storage and separation: Status and challenges, EnergyChem 1(1) (2019) 100006.

[19] Y.-S. Kang, Y. Lu, K. Chen, Y. Zhao, P. Wang, W.-Y. Sun, Metal-organic frameworks with catalytic centers: From synthesis to catalytic application, Coordination Chemistry Reviews 378 (2019) 262-280.

[20] Z. Hu, B.J. Deibert, J. Li, Luminescent metal-organic frameworks for chemical sensing and explosive detection, Chemical Society Reviews 43(16) (2014) 5815-5840.

[21] C.-Y. Sun, C. Qin, X.-L. Wang, Z.-M. Su, Metal-organic frameworks as potential drug delivery systems, Expert opinion on drug delivery 10(1) (2013) 89-101.

[22] H.-S. Wang, Metal-organic frameworks for biosensing and bioimaging applications, Coordination Chemistry Reviews 349 (2017) 139-155.

[23] S.-Y. Zhu, B. Yan, A novel sensitive fluorescent probe of S 2 O $82-$ and Fe 3+ based on covalent post-functionalization of a zirconium (iv) metal-organic framework, Dalton Transactions 47(33) (2018) 11586-11592.

[24] S. Halder, P. Ghosh, A. Hazra, P. Banerjee, P. Roy, A quinoline-based compound for explosive 2, 4, 6-trinitrophenol sensing: experimental and DFT-D3 studies, New Journal of Chemistry 42(11) (2018) 8408-8414. 
[25] M.J. Manos, E.J. Kyprianidou, G.S. Papaefstathiou, A.J. Tasiopoulos, Insertion of Functional Groups into a Nd3+ Metal-Organic Framework via Single-Crystal-to-SingleCrystal Coordinating Solvent Exchange, Inorganic chemistry 51(11) (2012) 6308-6314.

[26] L.-J. Zhang, L. Qi, X.-Y. Chen, F. Liu, L.-J. Liu, W.-L. Ding, D.-L. Li, G.-C. Yuan, J.Z. Tong, F.-Y. Chen, Synthesis, crystal structure and photophysical properties of two reduced Schiff bases derived from 5-Aminoisophthalic acid, Journal of Chemical Crystallography 49(4) (2019) 260-266.

[27] S. Aryanejad, G. Bagherzade, A. Farrokhi, Efficient and recyclable novel Ni-based metal-organic framework nanostructure as catalyst for the cascade reaction of alcohol oxidation-Knoevenagel condensation, Applied Organometallic Chemistry 32(2) (2018) e3995.

[28] R. Rani, A. Deep, B. Mizaikoff, S. Singh, Enhanced hydrothermal stability of Cu MOF by post synthetic modification with amino acids, Vacuum 164 (2019) 449-457.

[29] A. Kourtellaris, E.E. Moushi, I. Spanopoulos, C. Tampaxis, G. Charalambopoulou, T.A. Steriotis, G.S. Papaefstathiou, P.N. Trikalitis, A.J. Tasiopoulos, A microporous $\mathrm{Cu} 2+\mathrm{MOF}$ based on a pyridyl isophthalic acid Schiff base ligand with high CO 2 uptake, Inorganic Chemistry Frontiers 3(12) (2016) 1527-1535.

[30] J. Cheng, X. Xuan, X. Yang, J. Zhou, K. Cen, Preparation of a Cu (BTC)-rGO catalyst loaded on a Pt deposited $\mathrm{Cu}$ foam cathode to reduce $\mathrm{CO} 2$ in a photoelectrochemical cell, RSC advances 8(56) (2018) 32296-32303.

[31] S. Pramanik, C. Zheng, X. Zhang, T.J. Emge, J. Li, New microporous metal- organic framework demonstrating unique selectivity for detection of high explosives and aromatic compounds, Journal of the American Chemical Society 133(12) (2011) 4153-4155. 

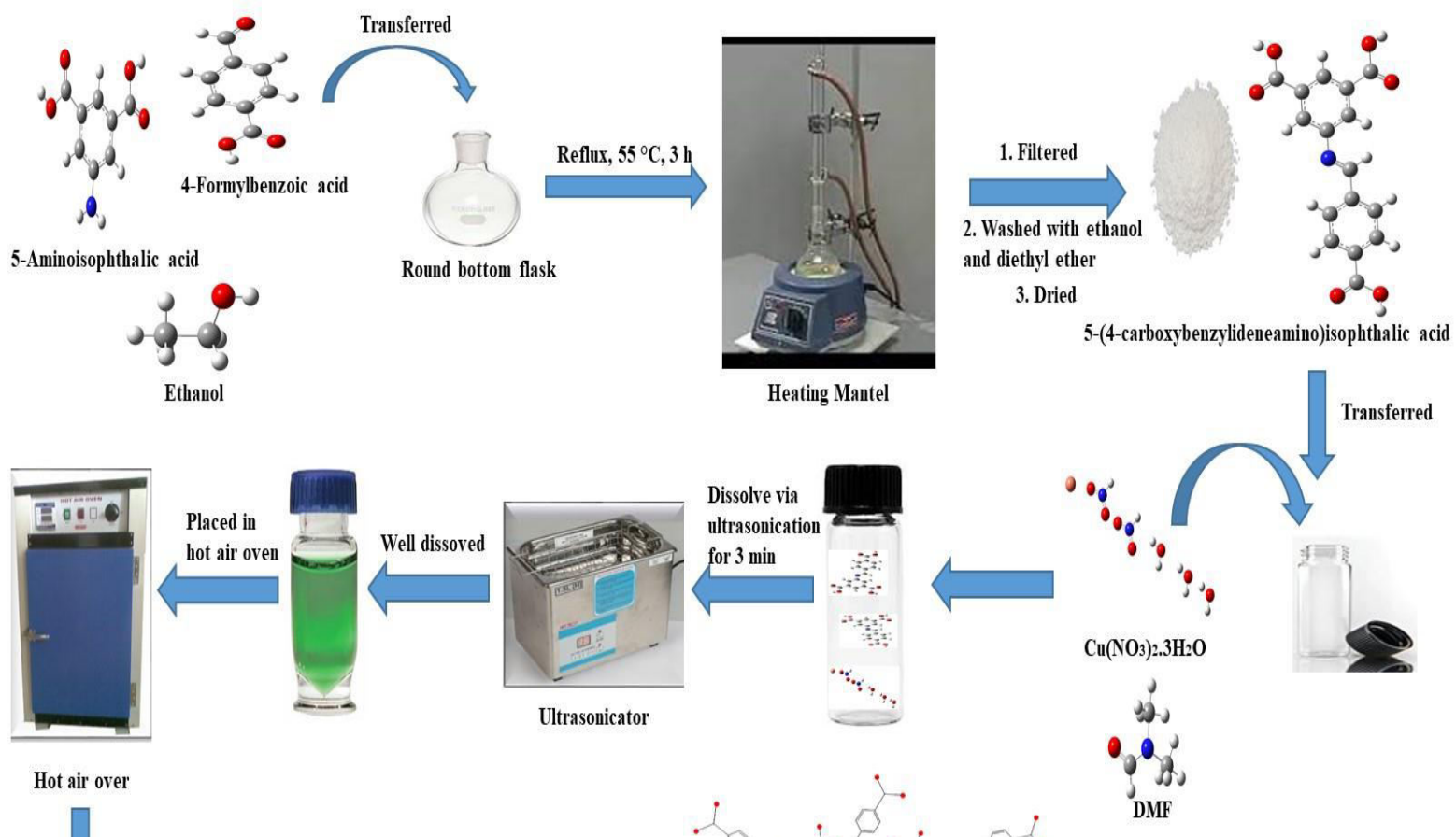

99
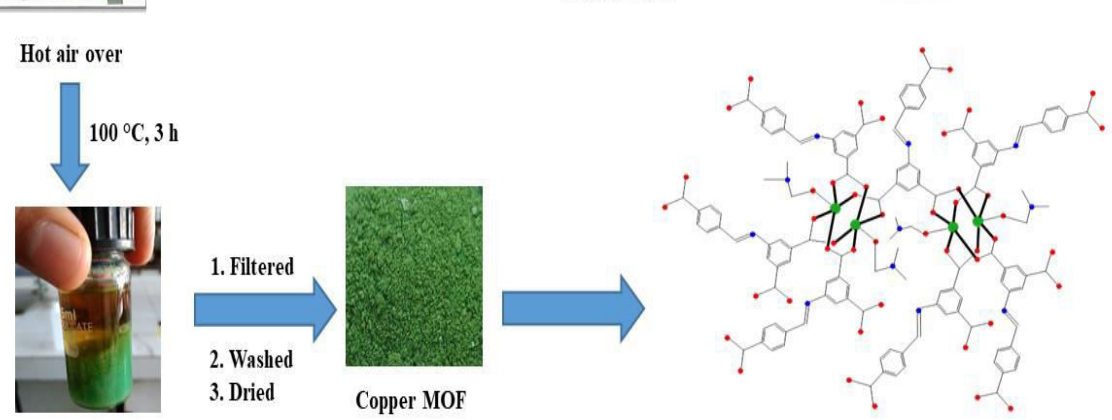

100 Scheme 1. Synthesis procedure for the preparation of imine functionalized copper MOF. 


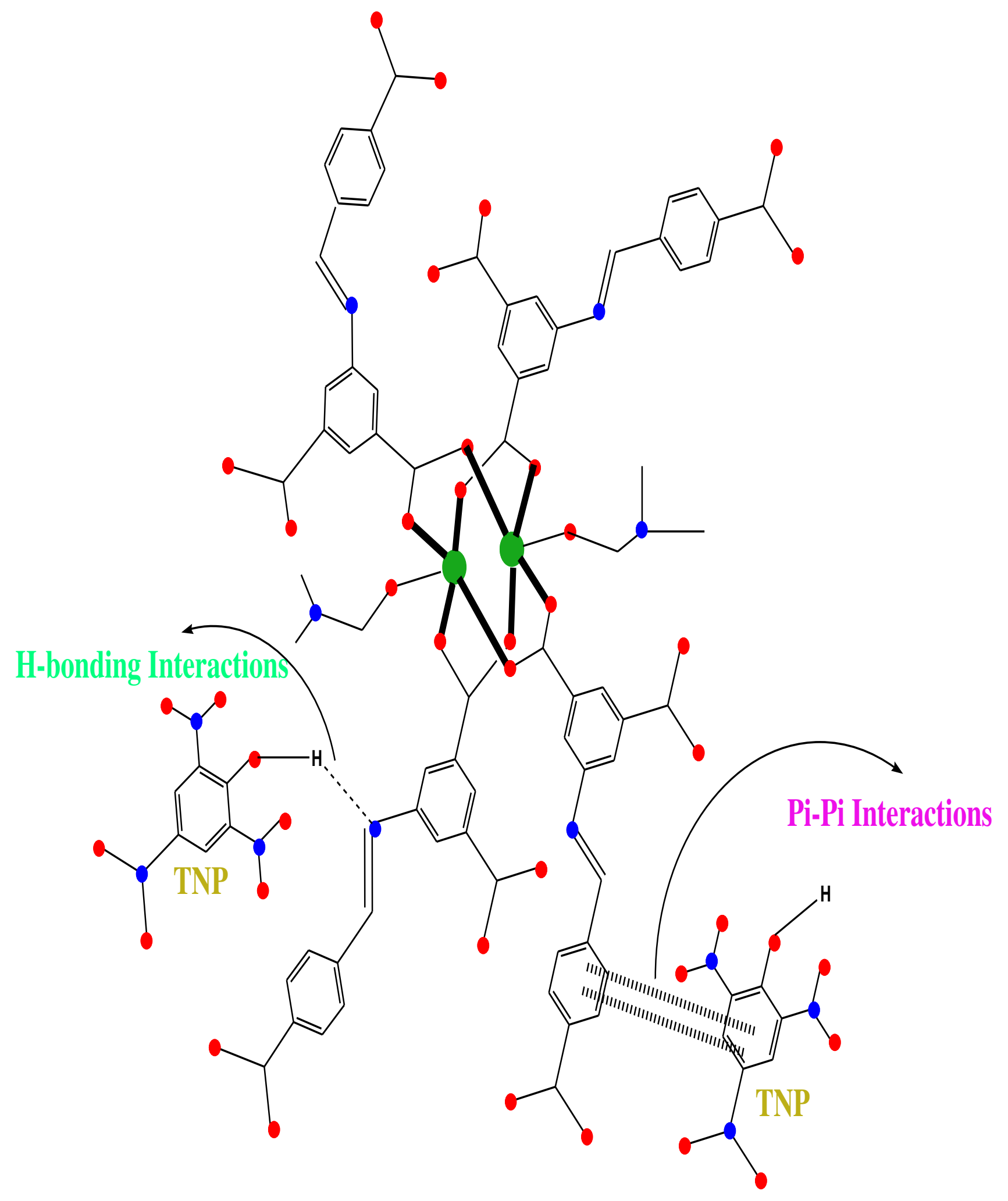

Scheme 2. Probable mechanism for TNP sensing by the imine functionalized MOF. 


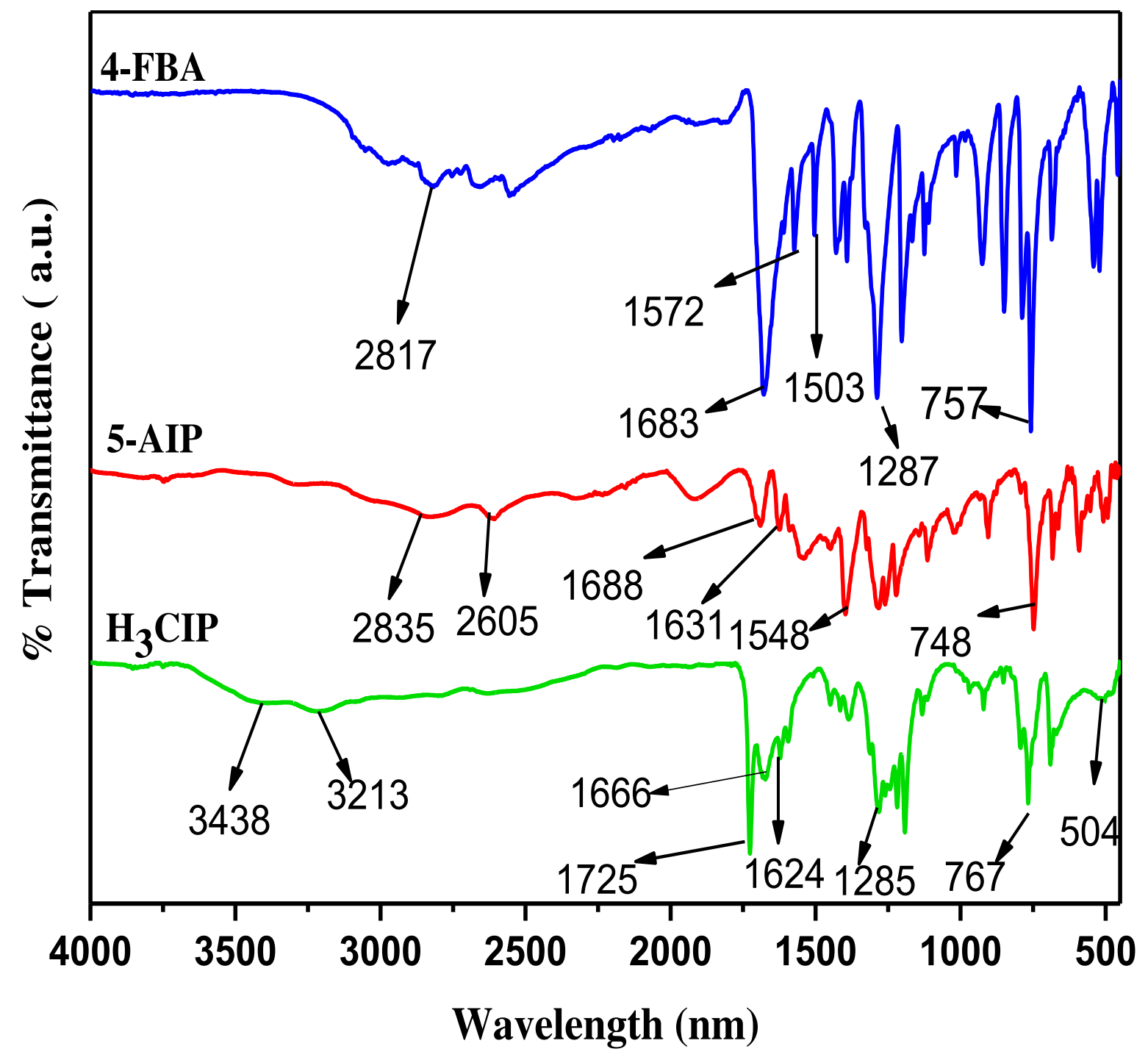




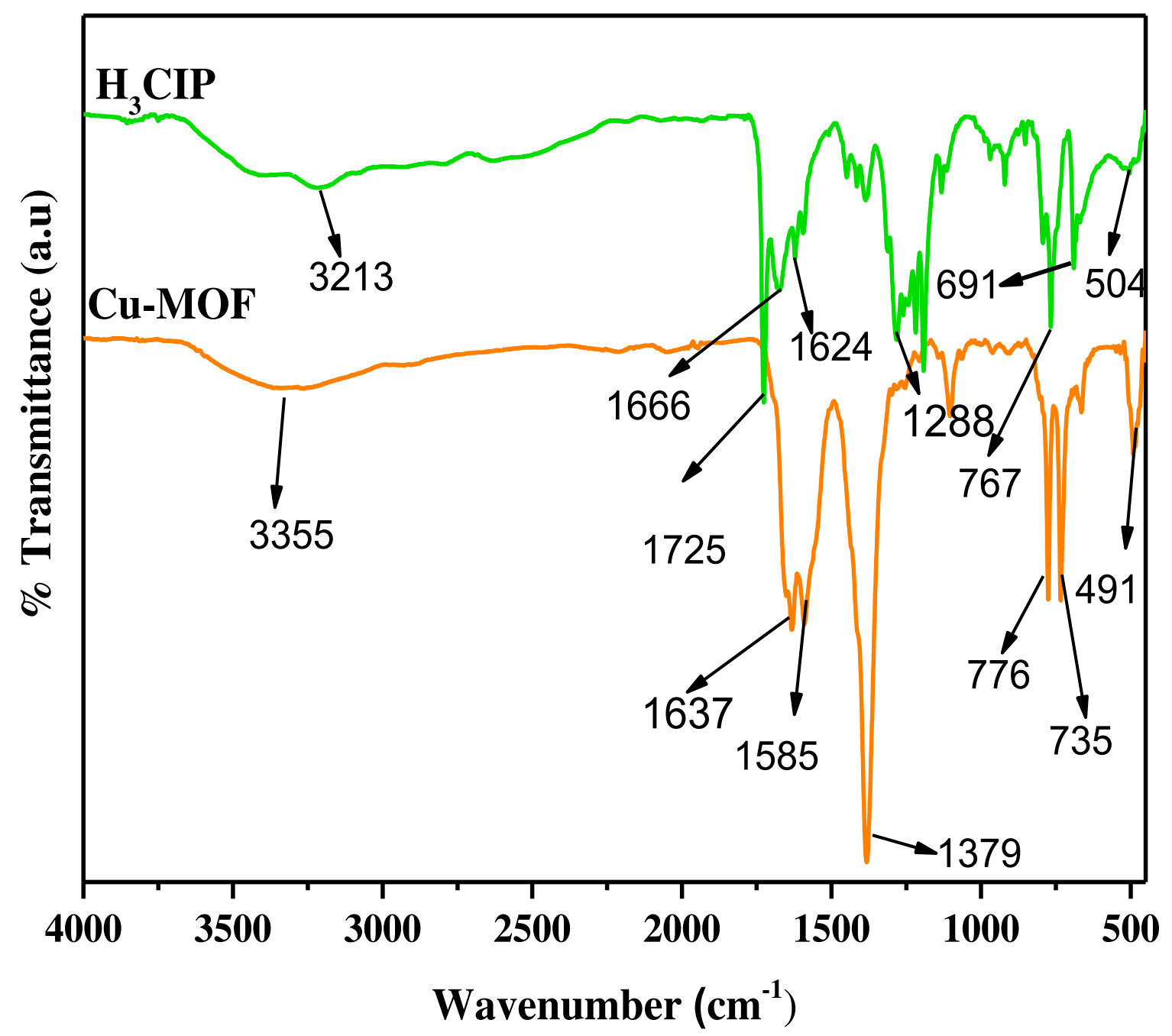

112 Figure 1. (a) FTIR spectra of the 4-FBA, 5-AIP, and product Schiff base ligand $\left(\mathrm{H}_{3} \mathrm{CIP}\right)$, (b)

113 FTIR spectra of the imine functionalized copper MOF. 


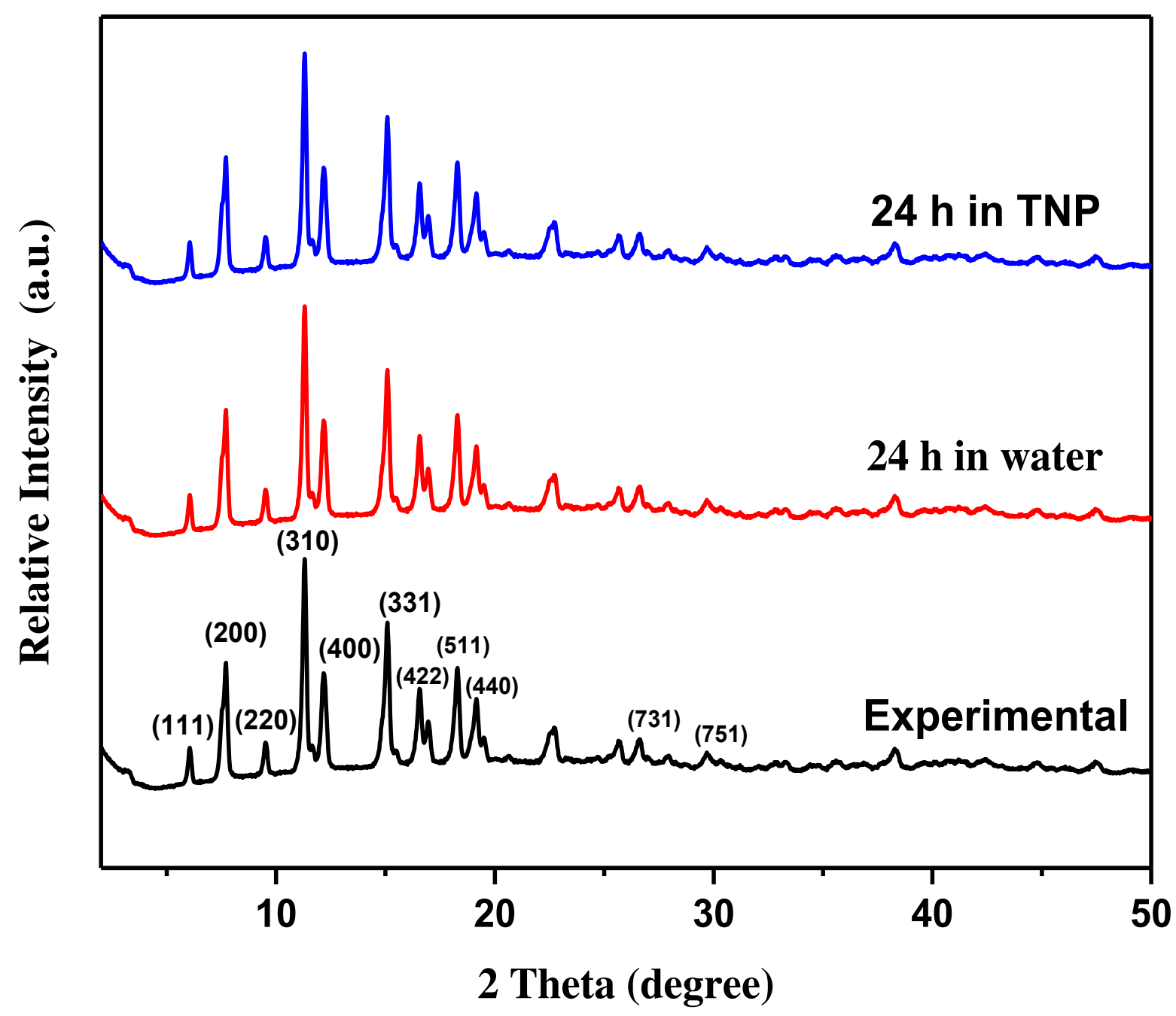

Figure 2. PXRD of Cu-MOF (experimental, water immersed and TNP immersed). The stability of $\mathrm{Cu}-\mathrm{MOF}$ in water as well as in aqueous TNP media were monitored by dispersing

117 them in the respective solvents for $24 \mathrm{~h}$, separation by centrifugation followed by washing 118 with methanol and drying at $80^{\circ} \mathrm{C}$ for $2 \mathrm{~h}$. 


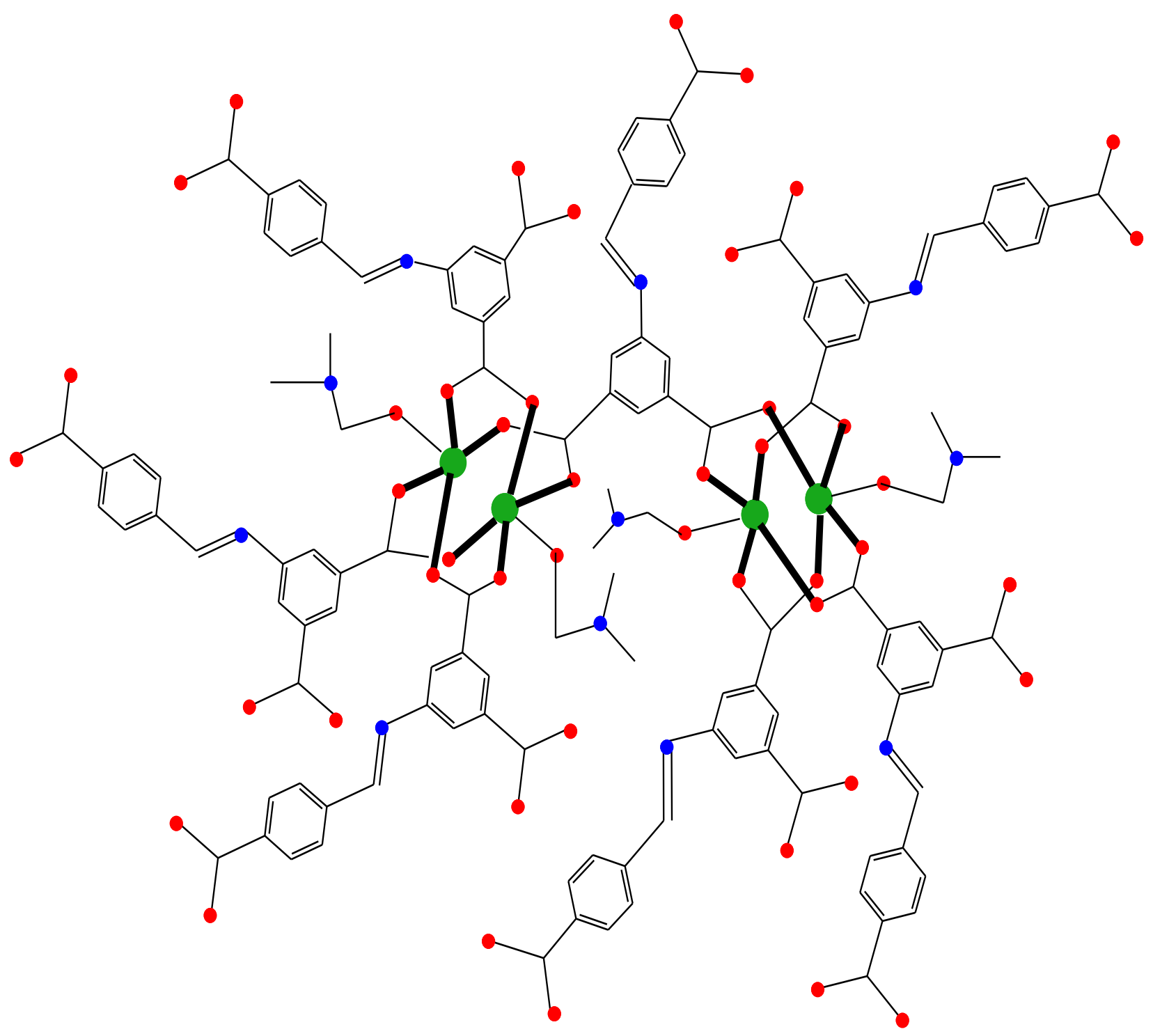

126 Figure 3. Representation of the proposed structure of the connectivity of the $\left[\mathrm{Cu}^{2+}\right]_{2}$ paddle 127 wheel SBUs through the $\mathrm{H}_{3} \mathrm{CIP}$ ligand in imine functionalized copper MOF. Colour code: $\mathrm{Cu}$ green, $\mathrm{O}$ red, $\mathrm{N}$ blue, $\mathrm{C}$ gray. $\mathrm{H}$ atoms are omitted for clarity. 

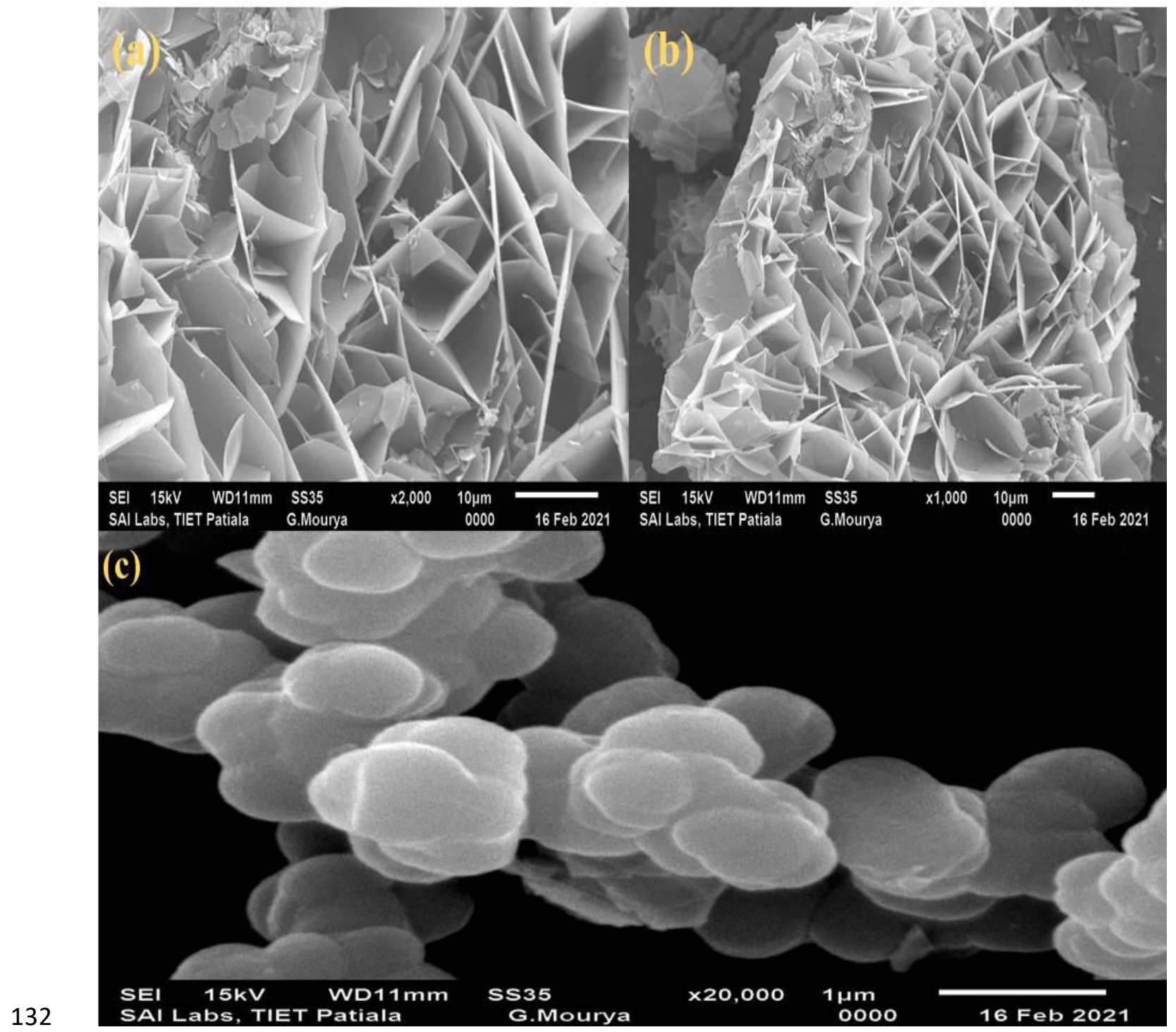
SAI Labs, TIET Patiala G Mourya 0000

133 Figure 4. SEM images of the synthesized $\mathrm{Cu}-\mathrm{MOF}$ with different magnification (a) $\mathrm{x} 1000$;

134 (b) $\mathrm{x} 2000 ;$ (c) $\times 20,000$. 


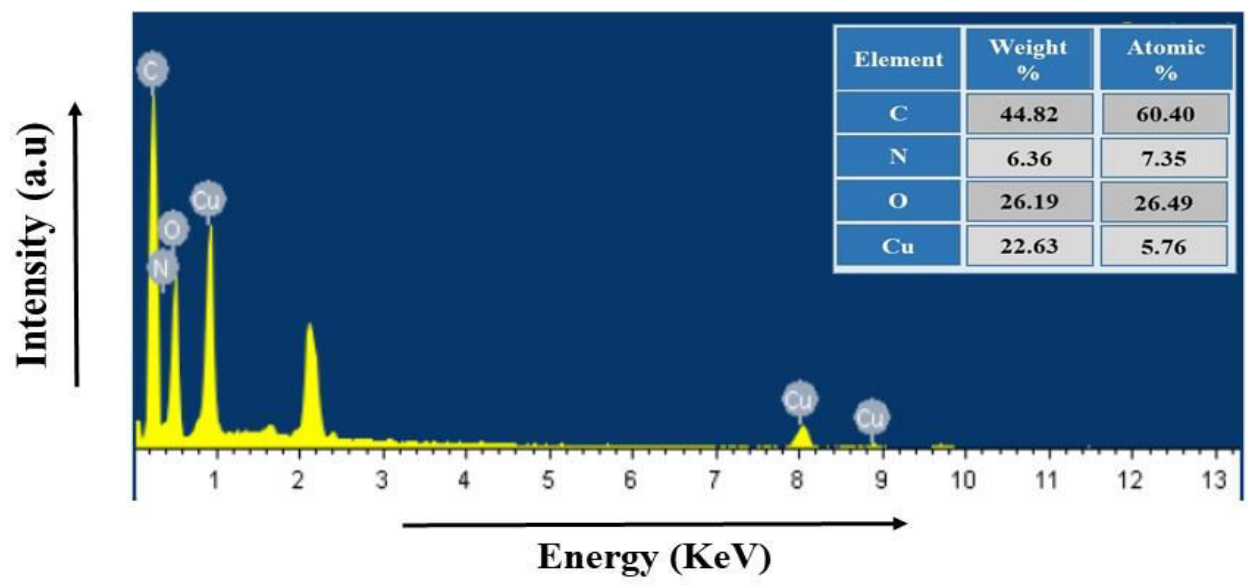

136

137 Figure 5. EDX spectra of imine functionalized copper MOF.

138

139

140

141

142

143

144

145

146

147

148

149 

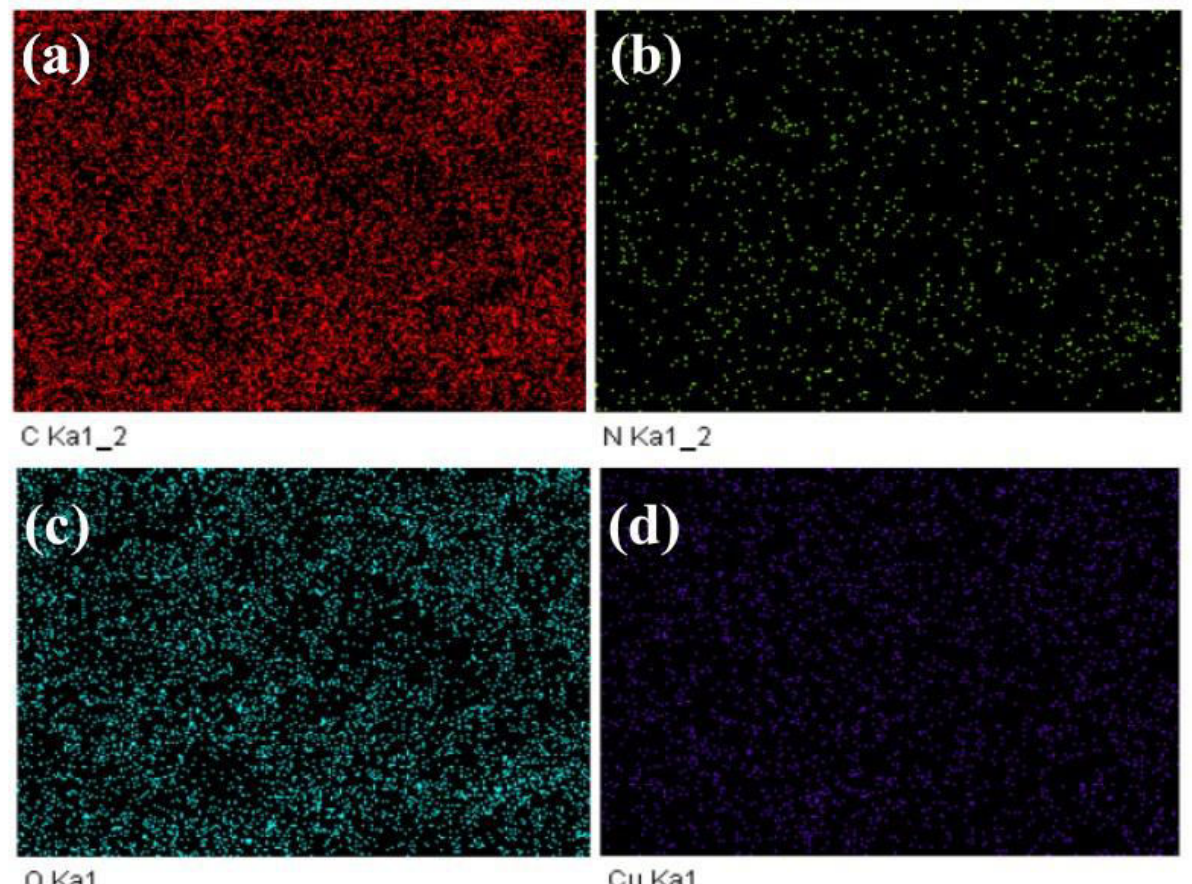

150 O Ka1

Cu Ka1

151 Figure 6. Elemental mapping of Cu-MOF showing (a) Carbon (C); (b) Nitrogen (N); (c)

152 Oxygen (O); (d) Copper $(\mathrm{Cu})$.

153

154

155

156

157 


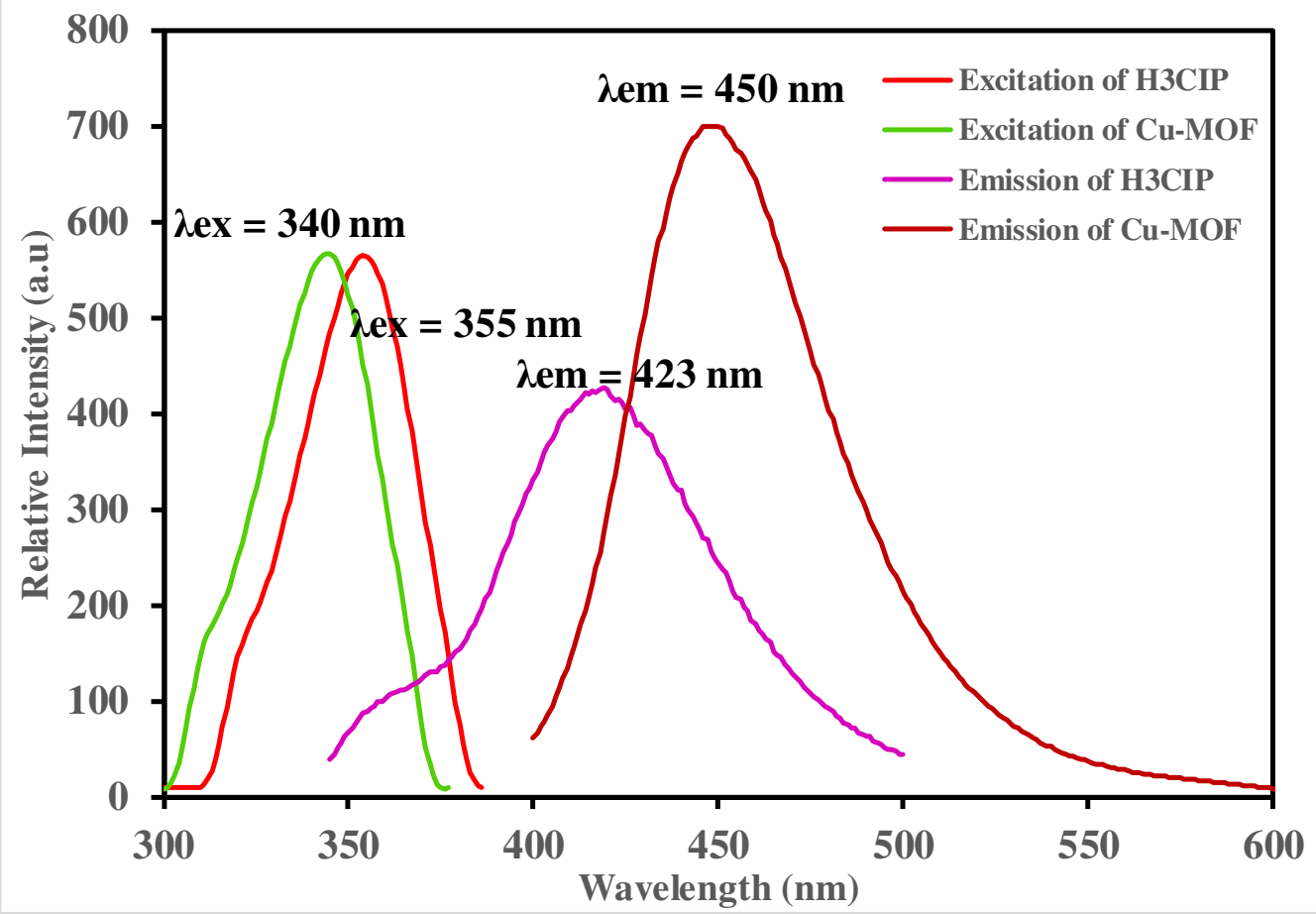

Figure 7. Room temperature excitation and emmision spectra of $\mathrm{H}_{3} \mathrm{CIP}$ and $\mathrm{Cu}-\mathrm{MOF}$ in the aqueous medium. 
(a)

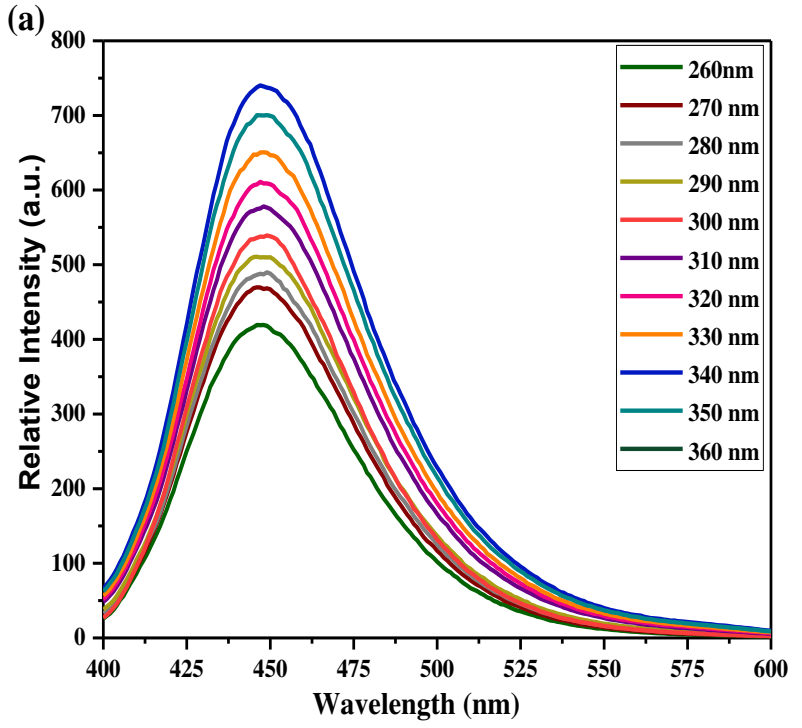

171

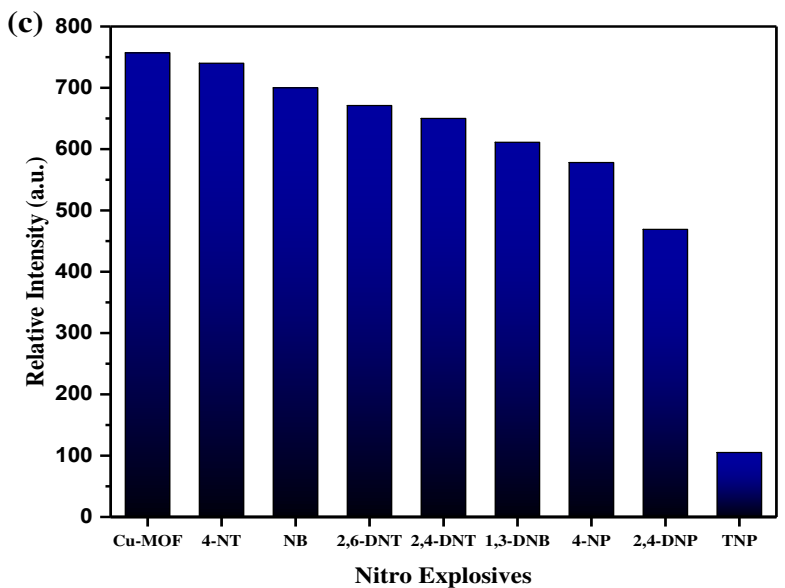

(b)

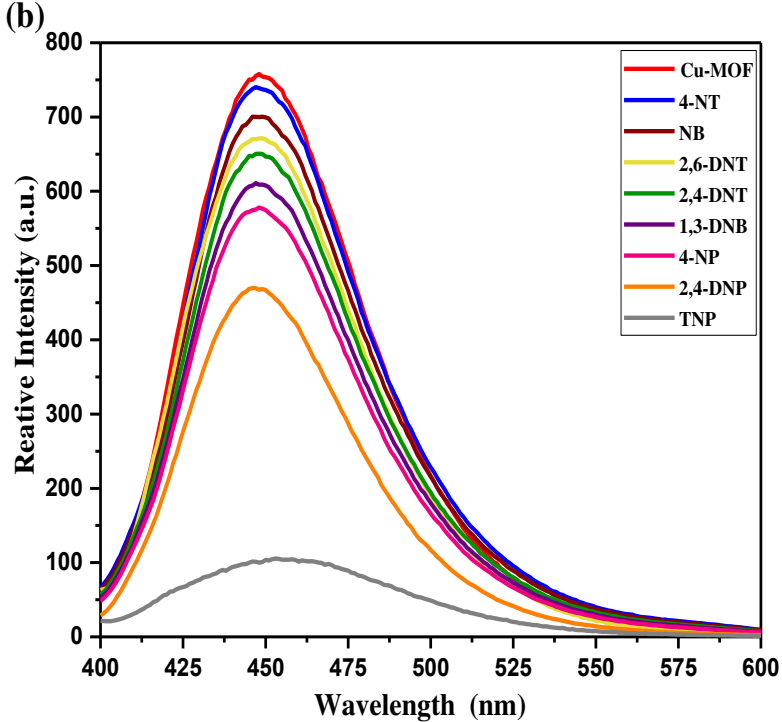

172

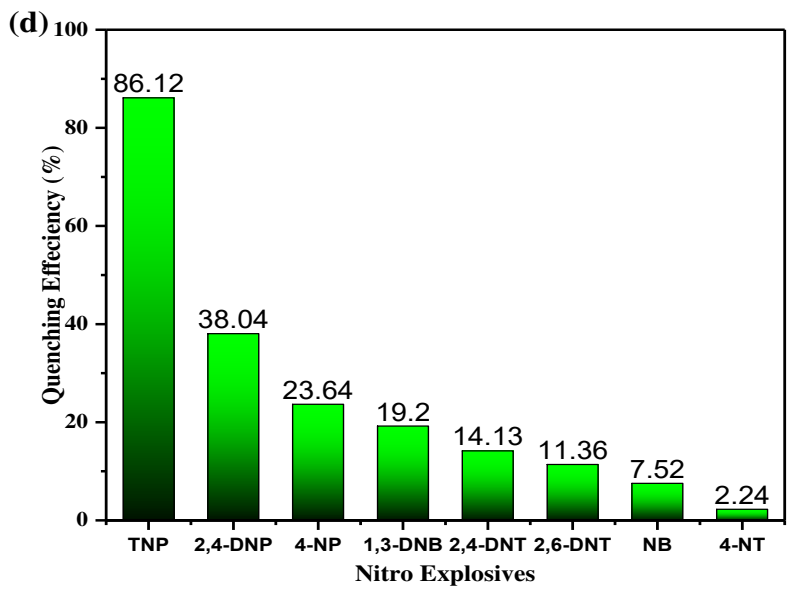

$174 \quad$ Figure 8. (a) Fluorescence emission spectra (excitation wavelength ( $\lambda$ ex) from 250 to $360 \mathrm{~nm}$ ) of

$175 \mathrm{Cu}-\mathrm{MOF}(1 \mathrm{mg})$ in aqueous solution $(3 \mathrm{~mL})$. (b) Suspension-state fluorescence spectra for $\mathrm{Cu}$ MOF (1 mg) dispersed in aqueous solution containing different nitro explosives $(20 \mu \mathrm{M}, 3 \mathrm{~mL})$ 
when excited at $340 \mathrm{~nm}$; (c) The relative intensities of emission peak monitored at $450 \mathrm{~nm}$ for Cu-

178

179

180

181

182

183
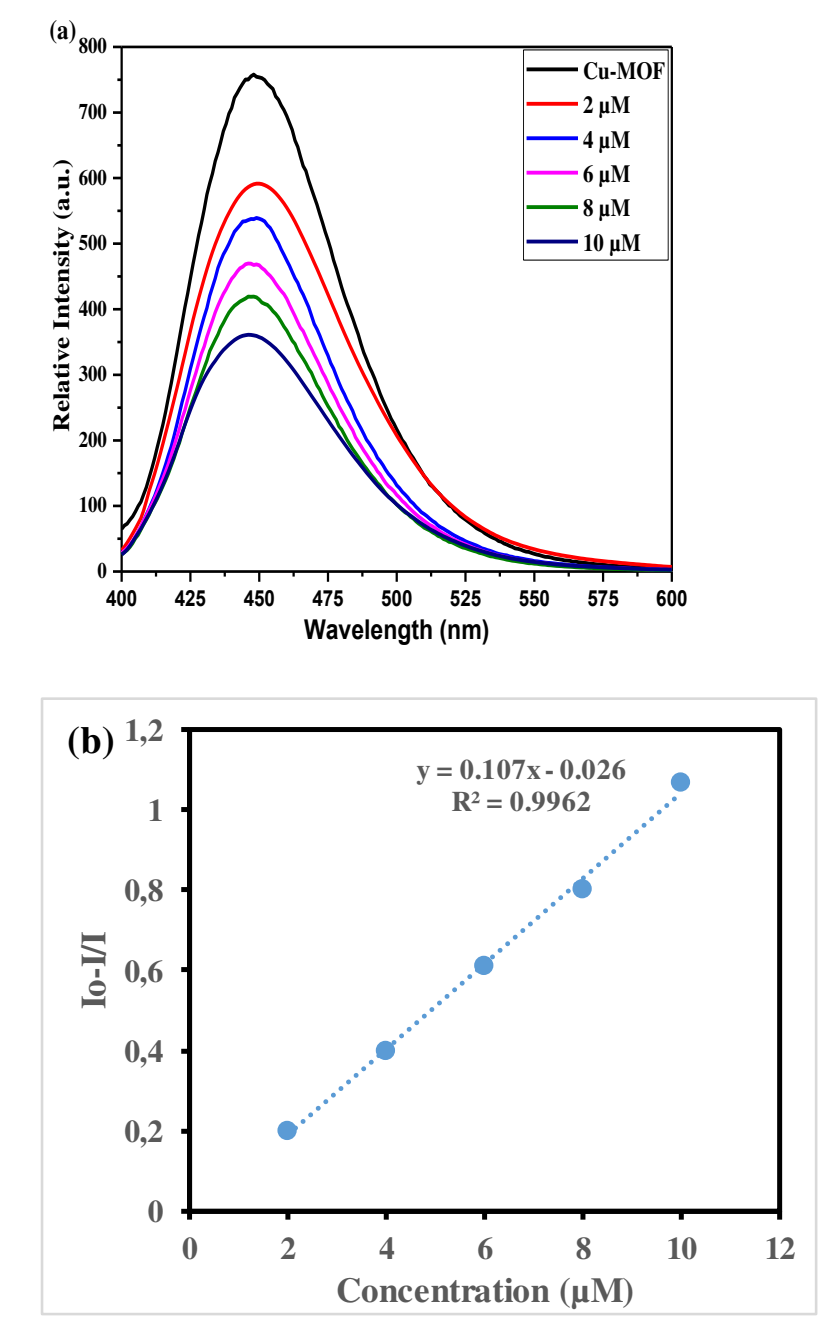

184

185

MOF dispersed in different nitro explosives aqueous solutions $(20 \mu \mathrm{M}, 3 \mathrm{~mL})$ when excited at 340 $\mathrm{nm}$. (d) Quenching efficiency of Cu-MOF $(1 \mathrm{mg})$ in the presence of aqueous solutions of various nitro explosives with the concentration of $(20 \mu \mathrm{M}, 3 \mathrm{~mL})$.

Figure 9. (a) Decrease in fluorescence emission of $\mathrm{Cu}-\mathrm{MOF}$ upon continuous addition of 0$10 \mu \mathrm{M}$ solution of TNP in aqueous medium. (b) Stern-Volmer plots of $\mathrm{I}_{0}-\mathrm{I} / \mathrm{I}$ versus TNP concentration in the range $0-10 \mu \mathrm{M}$ showing linear relationship and the fitting line in aqueous medium $\left(\mathrm{R}^{2}=0.9962 ; \mathrm{K}_{\mathrm{SV}}=1.07 \times 10^{4} \mathrm{M}^{-1}\right)$. 

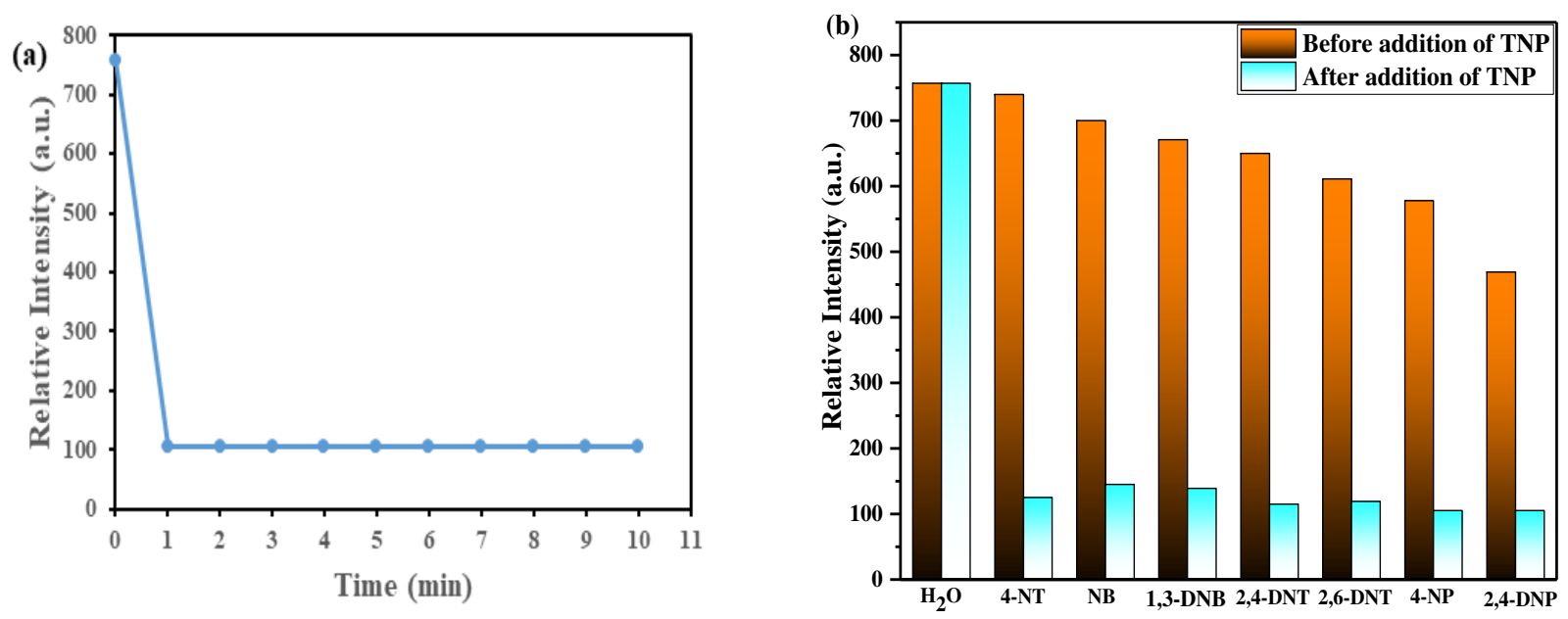

Figure 10. (a) Effect of response time on the fluorescent intensities upon the addition of aqueous solution of TNP $(20 \mu \mathrm{M}, 3 \mathrm{~mL})$ into the $\mathrm{Cu}-\mathrm{MOF}$ suspension. (b) Comparison of the luminescence intensity of $\mathrm{Cu}-\mathrm{MOF}(1 \mathrm{mg})$ upon the addition of TNP in the presence of other NAEs in aqueous solvent. The orange bars represent the emission of $\mathrm{Cu}-\mathrm{MOF}$ in the presence of $(20 \mu \mathrm{M}, 3 \mathrm{~mL})$ aqueous solution of NAEs. The sky blue bars represent the change of the emission that occurs upon the subsequent addition of $(20 \mu \mathrm{M}, 3 \mathrm{~mL})$ aqueous solution of TNP to a solution containing of $\mathrm{Cu}-\mathrm{MOF}$ and $20 \mu \mathrm{M}$ NAEs. 
205

206

207

208

209

210

211 (A)

(B) 


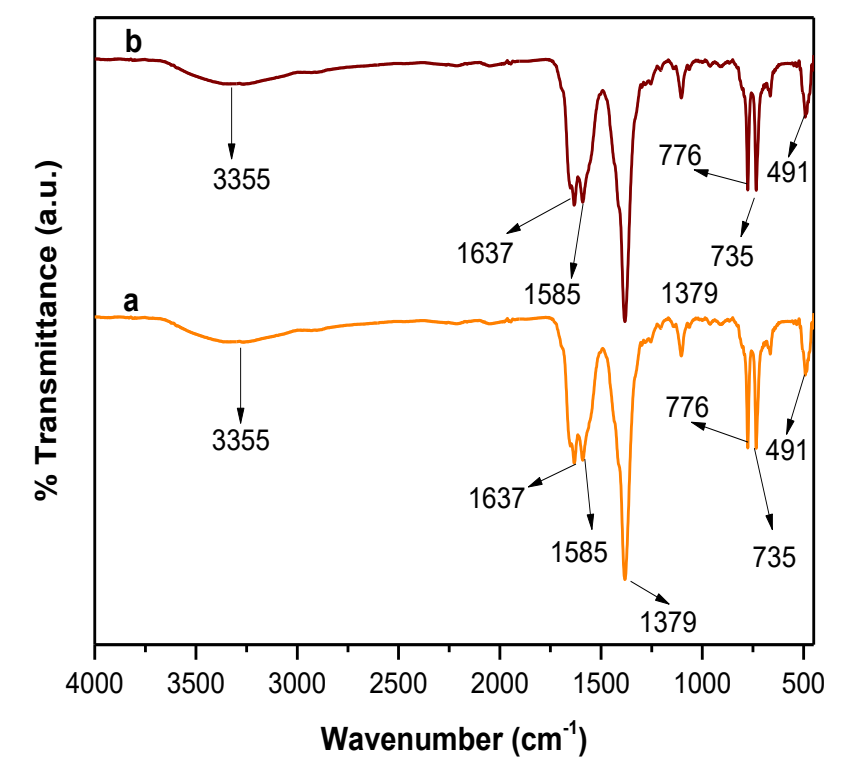

212

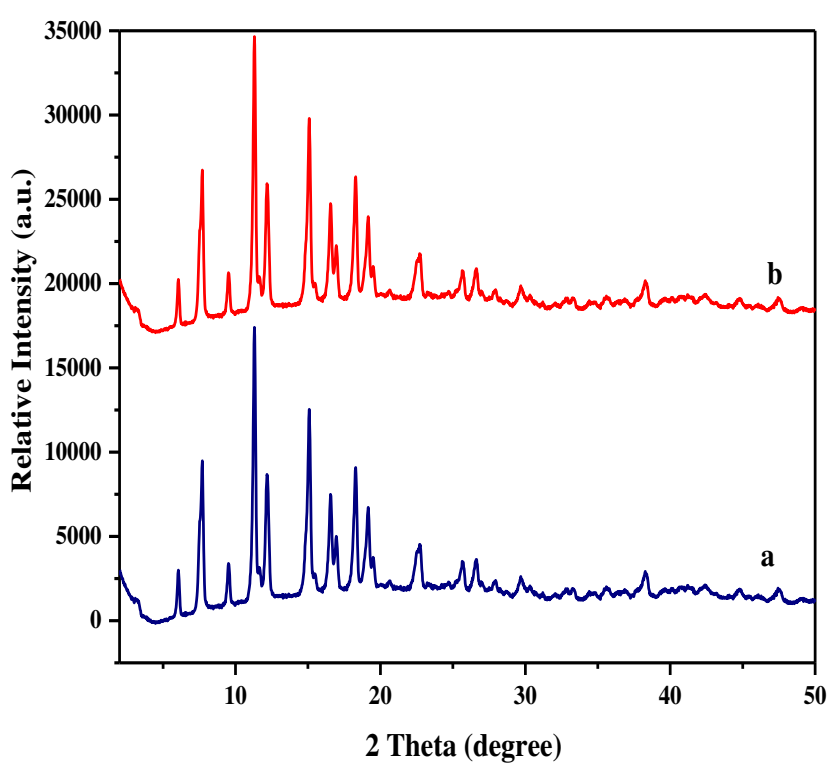

214 Figure 11. (A) FT-IR (B) PXRD pattern of (a) fresh Cu-MOF and (b) after TNP sensing in the 215 aqueous medium. 


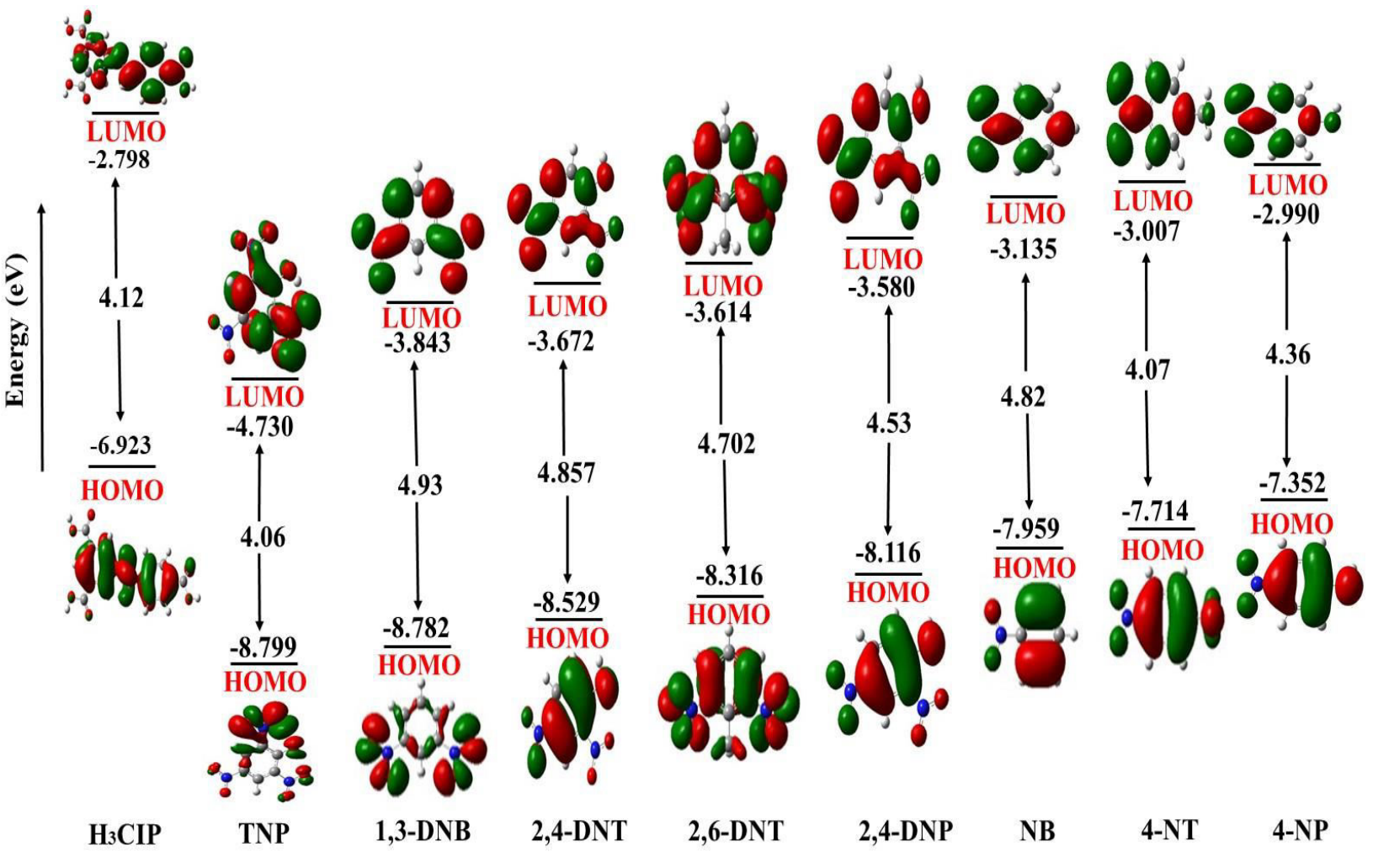

220 Figure 12. HOMO and LUMO plots and energy values (in eV) of various nitro explosives and

221 Schiff base fluorescent ligand $\left(\mathrm{H}_{3} \mathrm{CIP}\right)$. 


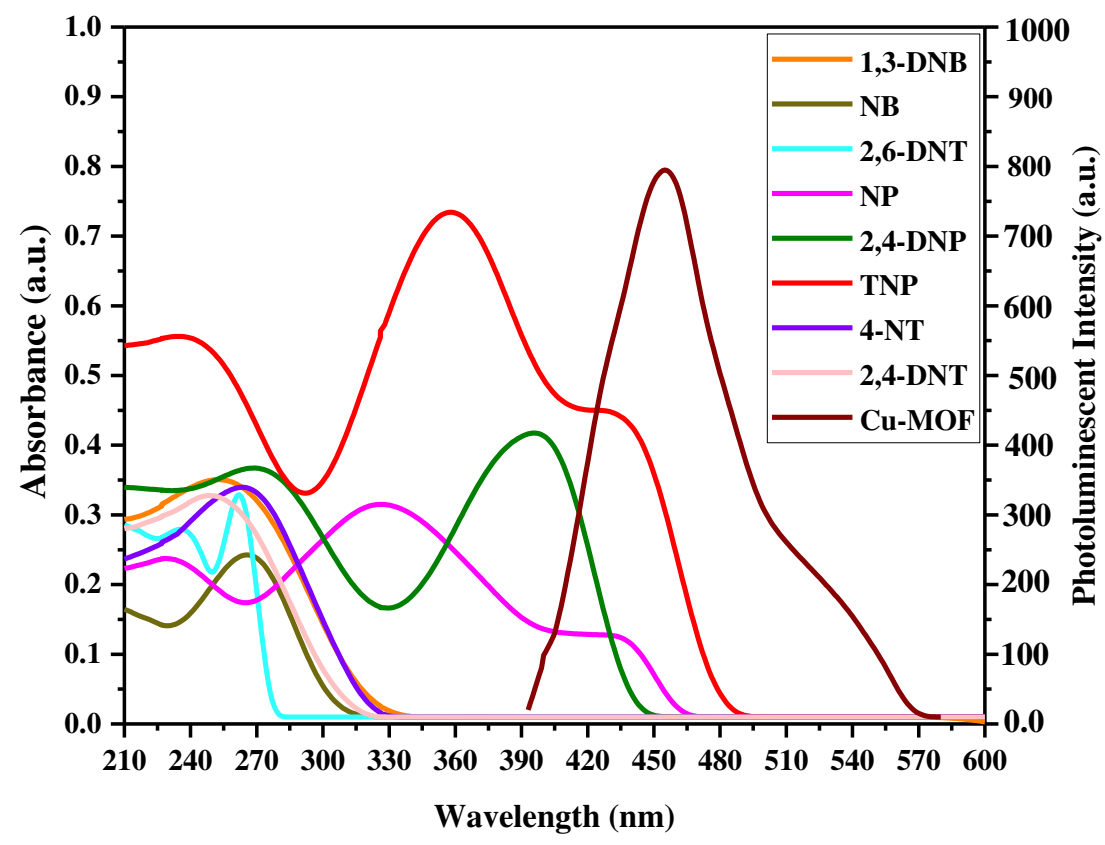

229 Figure 13. Spectral overlap of emission spectra of Cu-MOF and absorption spectra of TNP. 